Document downloaded from:

http://hdl.handle.net/10251/111713

This paper must be cited as:

Giovannelli, L.; Ródenas, J.; Navarro-Jiménez, J.; Tur Valiente, M. (2017). Direct medical image-based Finite Element modelling for patient-specific simulation of future implants. Finite Elements in Analysis and Design. 136:37-57. doi:10.1016/j.finel.2017.07.010

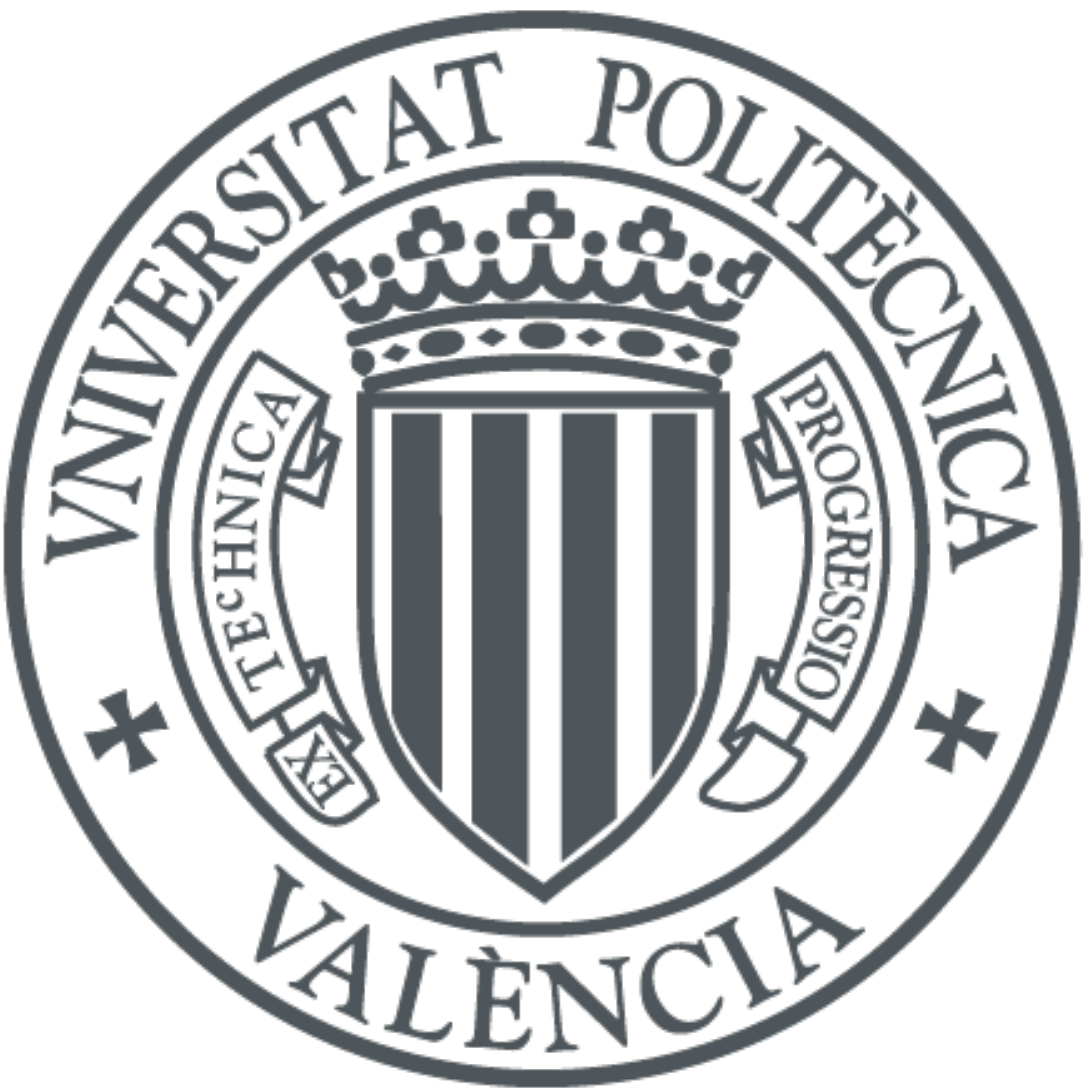

The final publication is available at

https://doi.org/10.1016/j.finel.2017.07.010

Copyright Elsevier

Additional Information 


\title{
Direct medical image-based Finite Element modelling for patient-specific simulation of future implants
}

\author{
L. Giovannellia,b,c ,J.J. Ródenas ${ }^{\mathrm{a}}$, J.M. Navarro-Jimenez ${ }^{\mathrm{a}}$, M. Tur ${ }^{\mathrm{a}}$ \\ ${ }^{a}$ Centro de Investigación en Ingeniería Mecánica (CIIM), \\ Universitat Politècnica de València, Edificio 5E, Spain \\ ${ }^{b}$ Grupo de Tecnología Sanitatia del IBV, CIBER de Bioingeniería, \\ Biomateriales y Nanomedicina (CIBER-BBN) \\ ${ }^{c}$ Instituto de Biomecánica de Valencia (IBV), \\ Universitat Politècnica de València, Edificio 9C, Spain
}

\begin{abstract}
In patient specific biomedical simulation, the numerical model is usually created after cumbersome, time consuming procedures which often require highly specialized human work and a great amount of man-hours to be carried out. In order to make numerical simulation available for medical practice, it is of primary importance to reduce the cost associated to these procedures by making them automatic. In this paper a method for the automatic creation of Finite Element (FE) models from medical images is presented. This method is based on the use of a hierarchical structure of nested Cartesian grids in which the medical image is immersed. An efficient $h$-adaptive procedure conforms the FE model to the image characteristics by refining the mesh on the basis of the distribution of elastic properties associated to the pixel values. As a result, a problem with a reasonable number of degrees of freedom is obtained, skipping the geometry creation stage. All the image information is taken into account during the calculation of the element stiffness matrix, therefore it is straightforward to include the material heterogeneity in the simulation. The proposed method is an adapted version of the Cartesian grid Finite Element Method ( $c g \mathrm{FEM}$ ) for the FE analysis of objects defined by images. $c g$ FEM is an immersed boundary method that uses $h$-adaptive Cartesian meshes non-conforming to the boundary of the object to be analysed.

The proposed methodology, used together with the original geometrybased $c g \mathrm{FEM}$, allows prosthesis geometries to be easily introduced in the
\end{abstract}


model providing a useful tool for evaluating the effect of future implants in a preoperative framework. The potential of this kind of technology is presented by mean of an initial implementation in $2 \mathrm{D}$ and $3 \mathrm{D}$ for linear elasticity problems.

Keywords: Cartesian grid Finite Element Method, Patient specific modelling, implant simulation, image based simulation

\section{Introduction}

Nowadays, the use of numerical models based on volumetric image data is widespread in biomechanics. Great efforts have been made to solve the elastic problem in a number of patient specific medical applications using the Finite Element Method (FEM), common in structural engineering. This is the case, for instance, of the prediction of bone fracture risk, Grassi et al. (2012), and the evaluation of bone quality parameters for the detection of osteoporosis, Alberich-Bayarri et al. (2008) and Roque et al. (2012). Preoperative implant simulation is a particularly promising use for patient-specific numerical models. This is fundamental for several applications such as studying the effect of positioning Bah et al. (2011), predicting the long term prosthesis performance thanks to the recent advances in bone remodelling Prez et al. (2014), designing customized implants taking advantage of optimization and 3D printing Sutradhar et al. (2016).

The most common procedures, Viceconti et al. (1998), to obtain numerical models from volumetric images can be broadly categorised into two groups: the voxel-based and geometry-based methods, both based on standard FEM. The former convert each voxel into an element of the FE mesh simplifying the modelling stage but typically providing problems with a high number of degrees of freedom, whereas the latter rely on modelling strategies, often hard to make automatic, to define geometrical domains from the image data which are then meshed as in standard FE. There are other aspects which can make one of the two methods more suitable than the other. For prosthesis analyses, for example, the geometry-based FE approach, Natali et al. (2006), is usually preferred because the assembly between the geometrical models can be performed with standard CAD tools. On the other hand, when a reliable relation between voxel values and elastic properties is available, as in the case of bone CT scan, it usually easier to take it into account using voxel-based methods due to the correspondence between elements and voxels. 
Doing the same with geometry-based meshes is more complicated because the elements do not conform to the pixels. A possible solution lies in assigning each integration point the average value of the stiffness corresponding to the surrounding pixels. Nevertheless the size of this influence area is not univocally defined, Müller-Karger et al. (2004).

New methods have recently been proposed in order to reduce both human intervention and computational cost in patient specific simulations. Most of them are extensions of geometry independent techniques defined by the umbrella term of Finite Elements in ambient space, Bordas et al. (2010) but available in the literature under a number of different names such as Fictitious Domain, Burman and Hansbo (2010), or Embedded Methods, Dolbow and Harari (2009), among others. These were originally developed to reduce the modelling effort for standard, CAD-based FE problems, which is, even in this case, responsible for about $80 \%$ of all the simulation time cost, Cottrell et al. (2009). These methods simplify the mesh generation by using an auxiliary domain $\Omega_{e}$, in general characterized by a simple, easy to mesh geometry, containing the problem domain $\Omega$. The auxiliary, or meshing, domain $\Omega_{e}$ is discretised instead of the problem domain $\Omega$. During the evaluation of the element integrals these methods require the information about the problem domain because the mesh does not conform to $\Omega$. In particular we remark the application to image-based problems of X-FEM, Legrain et al. (2011), Composite Finite Elements, Liehr et al. (2009), or Finite Cell Method (FCM), Ruess et al. (2012a).

We follow a similar path as the background of our proposal is the Cartesian grid Finite Element Method ( $c g$ FEM), Nadal et al. (2013), a method to solve CAD-based problems, which belongs to the family of the Finite Elements in ambient space. For the sake of clarity, we refer to it as geometrybased $\mathrm{cgFEM}$ in the following in order to distinguish it from the new method object of this paper which we call image-based $c g$ FEM and addresses the problem of solving patient specific numerical models from volumetric image data. Finally, we call FEAVox the Matlab-based code which implements both geometry-based and image-based cgFEM. In both its versions, this has points in common with the family of Finite Elements in ambient space, especially with the Finite Cell Method (FCM).

FCM is a powerful method which combines the fictitious domain approach with high-order hierarchical Ansatz spaces. The results, in the initial formulation of the FCM, are enhanced by using $p$-adaptivity, i.e. by increasing the order of the shape functions used for interpolation, while the spatial discreti- 
sation is kept uniform. An octree structure of subcell is used for integration. These coincide with the voxels image-based models and on a hierarchical octree structure in CAD-based models.

In geometry-based $\mathrm{cgFEM}$ the problem domain is immersed into a hierarchical structure of Cartesian grids. For the elements on the boundary the stiffness matrix is calculated solely by integrating the area of the element actually lying within the domain $\Omega$. Special techniques are used to account for the exact geometry during the element integration process thus avoiding modelling errors associated to an inexact representation of the boundary. A local $h$-adaptive refinement is used to enhance the solution.

In image-based $c g$ FEM, we superpose a coarse Cartesian mesh upon the bitmap. As in the FCM, each element contains an heterogeneous distribution of elastic properties. As a consequence, the element stiffness matrix integration carries out a kind of material homogenisation which makes it possible for $c g$ FEM to keep the number of degrees of freedom (DOF) lower than in classical voxel-based methods. We use $h$-adaptivity to tailor the mesh to the bitmap on the basis of the evaluation of the pixel value distribution in each element. This is meant to prevent excessive material homogenization and the resulting loss of accuracy in the solution.

\subsection{Objectives and paper structure}

The main contributions of this paper are:

- to present a cheaper and more parallelizable integration procedure for $\mathbf{k}^{e}$ based on the least-squares recovery of the Young's modulus field in each element and compare it with other integration schemes;

- to study some limitations in the mesh size due to the use of pixmaps and propose a cost-effective mesh $h$-adaptive method;

- to propose a method to combine geometry and image-based cgFEM perform patient-specific simulations of implants.

The next section opens briefly reminding the main features of geometrybased $c g$ FEM and follows describing its image-based version in detail and how to combine them to include CAD models into the medical image. The third section contains analyses of reference problems used for the validation of the method whereas in the fourth one the method is applied to real medical images in 2D and 3D to illustrate the behaviour of the proposal. Finally the conclusions are discussed in the fifth section. 


\section{The methodologies implemented in the software FEAVox}

\subsection{The method background: the geometrical cgFEM}

cgFEM was originally intended as an alternative to standard geometryconforming FEM. The geometry from a CAD model is intersected with a quadtree/octree-inspired hierarchical structure of Cartesian grids, see Figure 1 a) and b), and the calculation mesh is obtained by deleting the external elements. The remaining elements can be internal or be cut by the contour.

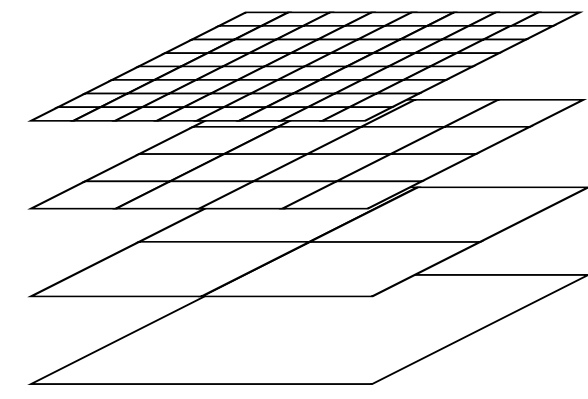

a)

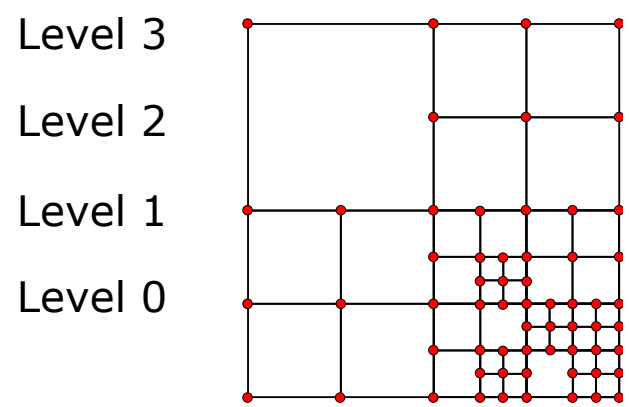

b)

Figure 1: $c g$ FEM mesher. a) First levels of the hierarchical structure of nested Cartesian grids; b) Example of non-conforming Finite Element mesh with $c g \mathrm{FEM}$

The former have proportional $\mathbf{k}^{e}$ matrices if they share the same material properties and are integrated as standard FE. The latter are integrated by decomposition in triangular integration subdomains, to take into account the non conformity of the interpolation integration domains, see Figure 2. In order to avoid geometrical approximation at this stage, in the case of NURBs based geometrical models, we use transfinite integration in 2D, Nadal (2014), or a NEFEM inspired integration technique in 3D, Marco et al. (2015).

On the one hand this guarantees a speed-up in the integration process because the complexity of the integration will be essentially associated to the dimensions of the boundary, i.e., a D-1 dimensional space (from 2D to 1D and from 3D to 2D) : as the elements in the bulk have proportional stiffness matrices the integration computational cost is only proportional to the number of elements laying on the boundary. On the other hand, the nested grid structure makes it possible to optimise the number of degrees of freedom by a fast mesh $h$-adapting process, and to reorder them hierarchically to reduce the computational cost, during the solving stage. For further details about 


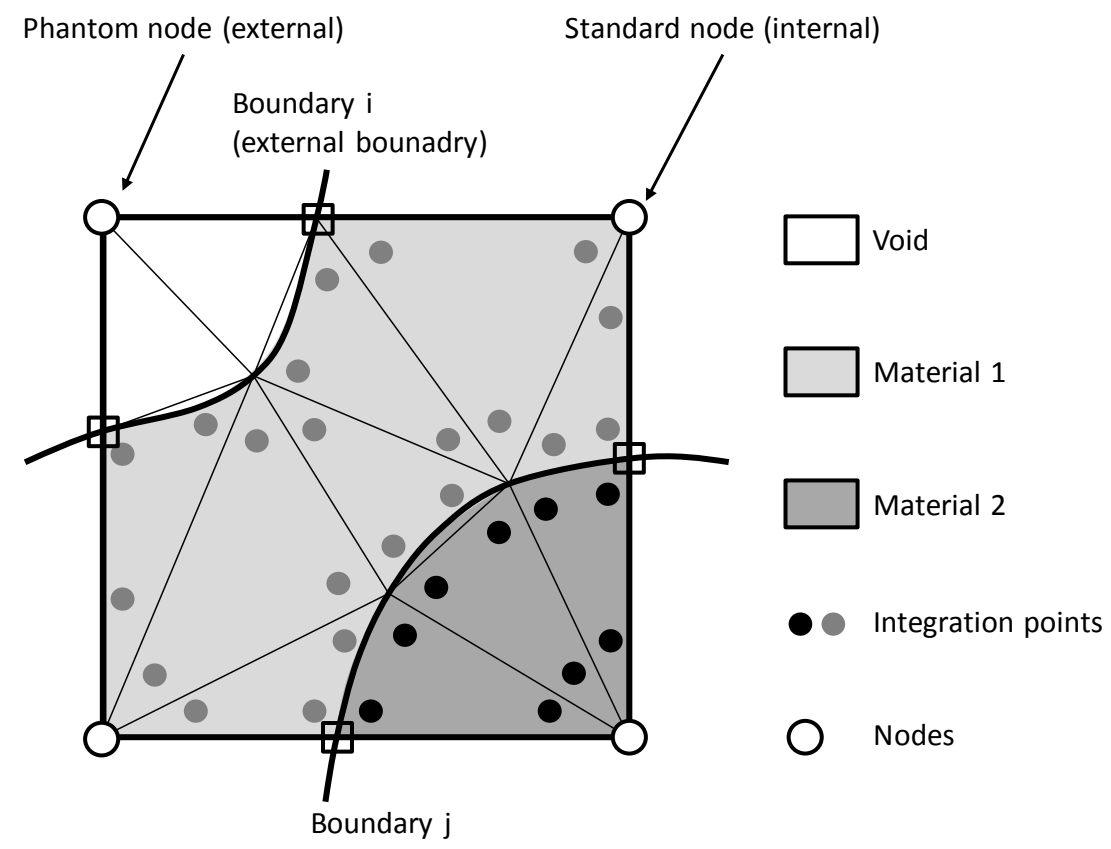

Figure 2: Triangular integration subdomains of $c g$ FEM element containing geometrical boundaries

the hierarchical structure-based reordering of the DOF in the FE system of equations, see Nadal (2014). In $c g$ FEM the mesh is obtained by an initial refinement based on geometrical criteria (the characteristics of the boundary portions contained in the elements on the boundary) and an iterative one guided by a Zienkiewicz and Zhu error estimator, Zienkiewicz and Zhu (1987) and, with an enhanced version of the superconvergent patch recovery (SPR) technique Nadal et al. (2015). Since the FE mesh is not conforming to the geometry the Dirichlet boundary conditions are enforced via stabilized Lagrangian multiplier techniques, Tur et al. (2014) and Tur et al. (2015).

The resulting stiffness matrix $\mathbf{k}^{e}$ of such an element homogenizes the elastic behaviour of the different domains. This is the procedure which inspires the element integration in image-based cgFEM. Geometry and image-based $c g$ FEM mainly differ on the integration method, but they share the strengths provided by the nested Cartesian grid structure: the fast meshing process and the hierarchical DOF reordering which increases the solver speed. 


\subsection{The image-based cgFEM}

The image-based $c g$ FEM follows a philosophy similar to the one used for the integration of the elements on the boundary in the geometry-based $c g$ FEM, whose resulting stiffness matrix $\mathbf{k}^{e}$ homogenizes the elastic behaviour of the different domains see Figure 2. This is the procedure from which the element integration in image-based cgFEM directly descends. After the creation of the proper Cartesian FE mesh, see Figure 3 a), the calculation of the element stiffness matrix $\mathbf{k}^{e}(1)$ homogenizes different elastic behaviours associated to the pixels on the basis of their value. Note that, following this approach, not only $\mathbf{B}$, the matrix containing the shape function derivatives in linear elasticity, depends on the spatial coordinates $(\mathbf{x})$ but also $\mathbf{D}$, the linear Hooke's law matrix, does. As a consequence, $\mathbf{k}^{e}$ is evaluated as:

$$
\mathbf{k}^{e}=\int_{V^{e}} \mathbf{B}^{T}(\mathbf{x}) \mathbf{D}(\mathbf{x}) \mathbf{B}(\mathbf{x}) d V
$$

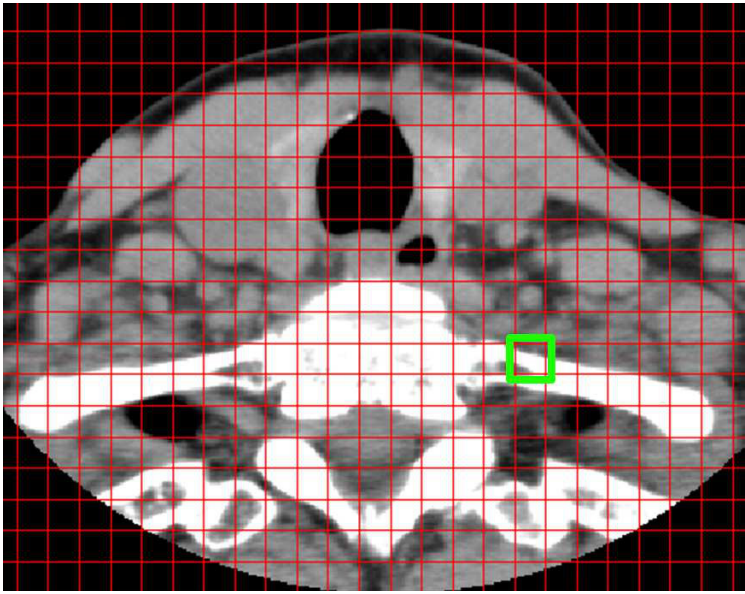

a)

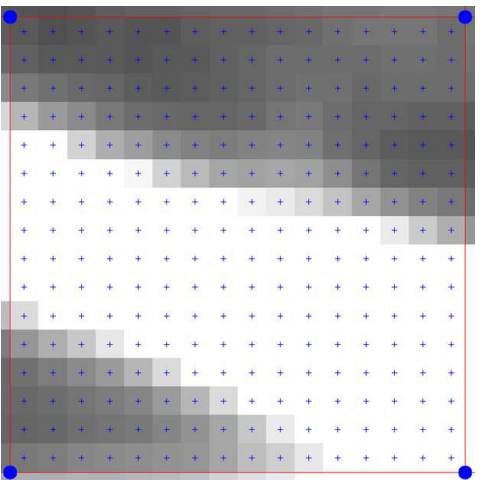

b)

Figure 3: a) Detail of neck CAT scan cross section immersed in uniform mesh. b) Magnification of the element highlighted in green

In the process all the pixels contained in the elements are taken into account at the integration stage. As opposite to the procedures used for this purpose in conforming meshes, in the Cartesian environment this is straightforward because, as explained later in this Section, the mesh creation guarantees that an integer number of pixels is contained in each element. The evaluation of the element integral is carried out by adopting a numerical quadrature rule, see (2), based,for example, on the Riemann sum in which 
one integration point is located at the centre of each pixel $i$, see Figure $3 \mathrm{~b}$ ), and the weight $w_{i}$, corresponding to each pixel/integration point is constant for all pixels and equal to the relative area of each pixel in the element.

$$
\mathbf{k}^{e}=\sum_{i=1}^{I P} \mathbf{B}^{T}\left(\boldsymbol{\xi}_{i}\right) \mathbf{D}\left(\boldsymbol{\xi}_{i}\right) \mathbf{B}\left(\boldsymbol{\xi}_{i}\right)\left|\mathbf{J}\left(\boldsymbol{\xi}_{i}\right)\right| w_{i}
$$

where $I P$ is the number of integration points in the element, i.e. the number of pixels in the element, and $|\mathbf{J}|$ is the determinant of the corresponding Jacobian matrix.

The Riemann sum approach shown in (2) has some limitations: in general, it is less accurate compared with a Gauss quadrature and elements belonging to different levels of the hierarchical mesh contain a different number of integration points, because a single integration point is associated to each pixel. The first consequence is the loss of accuracy of the numerical integration as the mesh is refined beyond a certain degree. In order to address the loss of accuracy of the integration strategy, an integration scheme similar to the one presented in Yang et al. (2012) was used. This consist of decomposing each element in a number of integrations subdomains, $I S$, corresponding to the pixels domains. In each of them the material properties are considered constant and the proper Gaussian quadrature of $I P$ integration points is used.

$$
\mathbf{k}^{e}=\sum_{i=1}^{I S} \sum_{j=1}^{I P} \mathbf{B}^{T}\left(\boldsymbol{\xi}_{i j}\right) \mathbf{D}_{i} \mathbf{B}\left(\boldsymbol{\xi}_{i j}\right)\left|\mathbf{J}\left(\boldsymbol{\xi}_{i j}\right)\right| w_{j}
$$

This prevents the integration error form increasing during the mesh refinement at the expense of a higher computational cost for the calculation of the $\mathbf{k}^{e}$ matrices. In both schemes, the Riemann sum and the decomposition in integration subdomains, the overall number of integration points is constant and independent from the mesh but the latter requires more integration points than the Riemann Sum scheme.

The second feature the Riemann sum scheme shares with the decomposition in subdomains approach is that, since they have a different number of integration points, the computational procedures are harder to parallelise. The solution proposed is a least squares based integration scheme. The material property samples provided by the image are fitted in each element by 
the polynomial $\mathbf{p}(\boldsymbol{\xi})^{T} \mathbf{a}_{e}$ where the column vector $\mathbf{p}$ is a polynomial basis $\mathbf{p}=\left\{1 \xi \eta \xi^{2} \xi \eta \eta^{2} \ldots\right\}^{T}$ of a prescribed order and the column vectors a the polynomial coefficients for each element $e$. The resulting material property field is piecewise continuum and, in general, $C_{0}$ discontinuous at the interfaces between elements. The polynomial $\mathbf{p}(\boldsymbol{\xi})^{T} \mathbf{a}_{e}$ can be least squares fitted to the material properties $P_{i}$ at the centre of the pixels $\boldsymbol{\xi}_{i}$ by the minimization of the functional $\Pi$ with respect to each one of the polynomial coefficients $a_{j}$ as follows:

$$
\min _{\alpha_{j}} \Pi=\min _{\alpha_{j}} \sum_{i}^{N P}\left(P_{i}-\mathbf{p}\left(\boldsymbol{\xi}_{i}\right)^{T} \mathbf{a}\right)^{2}
$$

This leads to the system of linear equations

$$
\left[\sum_{i}^{N P} \mathbf{p}\left(\boldsymbol{\xi}_{i}\right) \mathbf{p}\left(\boldsymbol{\xi}_{i}\right)^{T}\right] \mathbf{a}=\sum_{i}^{N P} P_{i} \mathbf{p}\left(\boldsymbol{\xi}_{i}\right)
$$

which has to be solved to compute the coefficient column vector a for each element in the mesh. For a visual representation of the three methods see Figure 4 .

Finally the element stiffness matrix is computed with (2) using Gaussian quadrature required by the polynomial degree of the integrand. This reduces the computational effort necessary to calculate the $\mathbf{k}_{e}$ matrices because same set of $\mathbf{B}$ matrices, obtained for an element of an arbitrary level, can be scaled and used for all the elements in the mesh, Nadal et al. (2013).

It is worth highlighting that, in the framework of $h$-adaptivity, in which $c g$ FEM meshes consists of elements from different levels, this feature makes the LS technique more efficient than the IS and Riemann sum-based integration schemes. Independently from the overall number of IP in the mesh, these techniques require the full calculation of a $\mathbf{B}$ matrix for each Cartesian grid level involved in the mesh, which is particularly expensive in the case of coarse elements containing a high number of integration points.

For instance, if a mesh consists of elements from the level 4 of the Cartesian grid structure and each element contains $32 \times 32 \times 32$ pixels, the Riemann sum requires the $\mathbf{B}$ matrix to be computed at 32768 positions. In the case of linear interpolation functions, the number of locations is 8 times higher if the integration is carried out by using the IS techniques whereas, if LS technique is used, they are only 27. 
If the mesh is $h$-refined and also consists of level 5 elements of $16 \times 16 \times 16$ pixels the number of locations at which the $\mathbf{B}$ matrix has to be computed increases by 4096. Additional elements from the levels 6,7 and 8 introduce 512, 64 and 8 positions more respectively. In this case the total number of B matrices to compute is 37448 in the case of the Riemann sum integration techniques, whereas in the case of the LS techniques it continues to be 27 no matter the levels involved.

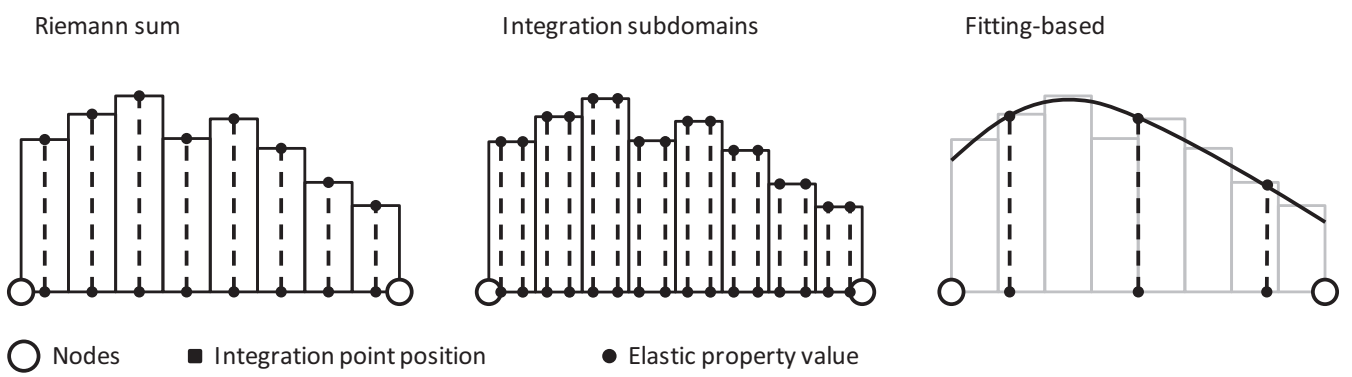

Figure 4: Schematic 1D representation for the integration techniques presented in Section 2.2 .

A drawback of the least square fitting is that it alone cannot guarantee neither the stability of the method nor the compliance with the energy conservation principles, as the values of the elastic properties extrapolated at the Gauss Points might not lay in a physically admissible range. In this paper we assume isotropic material models which only require two independent parameters such as the Young's modulus, which has to be positive, and the Poisson's ratio, which has to lay between 0 and 0.5 . To keep the the solution unique and physically consistent, we detect the elements which show unacceptable values of the elastic properties at the Gauss Points and scale the coefficients of the polynomial interpolation imposing that the values of elastic constants are in a consistent range and their mean value over the element is the same as in the uncorrected LS fitting.

In the following, we only use first order polynomial interpolations of the element elastic properties as a series of drawbacks appear for higher orders. It is worth anticipating that, for the interpolation of the FE solution, we use linear shape functions because polynomial order higher than 1 suffer important limitations in the framework of image-based $c g$ FEM, as it is made clear in Section 3. As the displacement is of low order, no gain is to be expected 
from increasing the order of the material property LS fitting. Instead it is counter-productive as the computational cost increases due to a) the higher number of IP and the corrections necessary to guarantee the method's stability, as more and more unrealistic elastic property values appear at the Gauss points due to the typical oscillatory behaviour of higher order polynomials.

In CT-scans the pixel values, expressed in Hounsfield scale, depend on the the average local X-ray absorption rate of the volumes represented by the pixels. In the literature, Dance et al. (2014) the range of Hounsfield values are related to different kinds of biological materials. For bones in particular, a number of results have been published which relate the Hounsfield scale to bone mineral density and finally to elastic material properties, usually through non linear relationships This aspect lies beyond the purpose of this paper, as a consequence, in the following, we will equally use different scales with $2 \mathrm{D}$ and $3 \mathrm{D}$ images without pretension of proposing reliable material properties but focusing on the numerical aspect of the method. Therefore, for simplicity, in the numerical examples, the relation between the pixel values and material properties will be defined as piecewise linear and interpolated from typical values from the literature and only the case of isotropic materials will be considered. The process originates relations between elastic properties and pixel values qualitatively similar to the schematic graph in Figure 5.

In order to avoid a too aggressive homogenisation of the local properties, the size of the FE mesh is tailored to the elastic property heterogeneity associated to the bitmap. In the hierarchical structure, the grid of the $n^{\text {th }}$-level is obtained by splitting each element of the grid of the $(n-1)^{\text {th }}$-level in two new elements in each direction, therefore, in the following level, each element generates $2^{D}$ new elements, where $D$ is the problem spatial dimension.

The image is immersed into an initial uniform grid in which the grey value distribution is evaluated, see Figure 8 a). This is the beginning of a recursive procedure of evaluation and $h$-refinement which stops when each element satisfies the limitation imposed by the user on the elastic property distribution or when the maximum mesh level allowed is reached, see Figure $8 \mathrm{~b})$.

Let $\alpha_{i}^{A}$ be the value of the Young's modulus assigned to the pixel $i$ within the domain $A$. This initial implementation is based on the evaluation of the $I_{R}^{e}$ index, which represents the relative elastic property range associated to the pixels in each element $e$ and is evaluated as: 


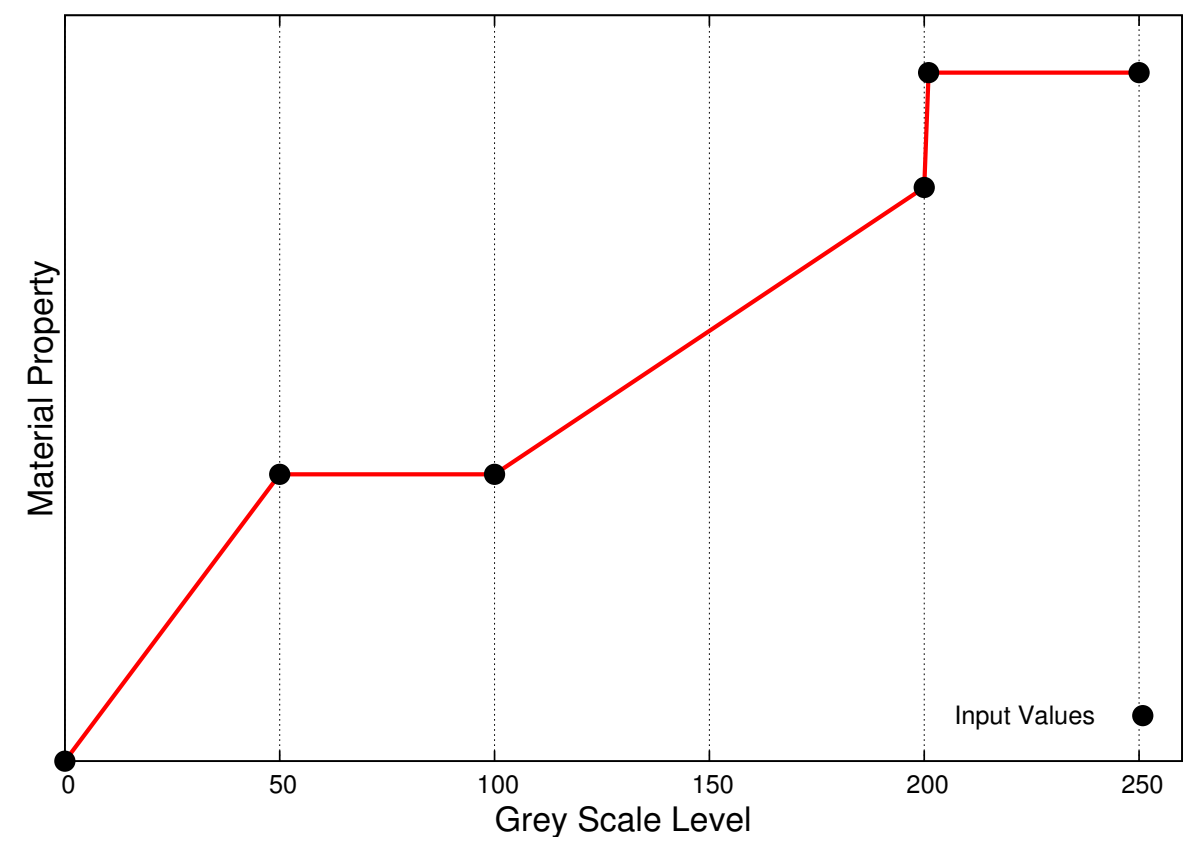

Figure 5: Schematic representation of the relation between grey scale pixel values and material properties

$$
I_{R}^{e}=\frac{\Delta \alpha^{\Omega^{e}}}{\Delta \alpha}=\frac{\max \left(\alpha_{i}^{\Omega^{e}}\right)-\min \left(\alpha_{i}^{\Omega^{e}}\right)}{\max \left(\alpha_{j}^{\Omega}\right)-\min \left(\alpha_{j}^{\Omega}\right)} ;
$$

The extension of the variability evaluation to all the constants ( 2 for isotropic or 21 for anisotropic and freely oriented orthotropic materials), required for the definition of the elastic compliance tensor, is straightforward. Nonetheless, for simplicity, all the meshes presented in this paper are refined on the basis of the Young's modulus only. This simplification is admissible as we are mainly interested in bone mechanics. A common assumption in this field is that of isotropic material with a constant value of the Poisson's ratio of 0.3 . The vast majority of relationships between elastic behaviour and Hounsfield values available in the literature only involves the Young's modulus value.

The outcome of range-based mesh refinement is highly problem-dependent and is affected by both the image characteristics and the voxel value-elastic property relation. In the numerical examples in Section 3 we use a value 


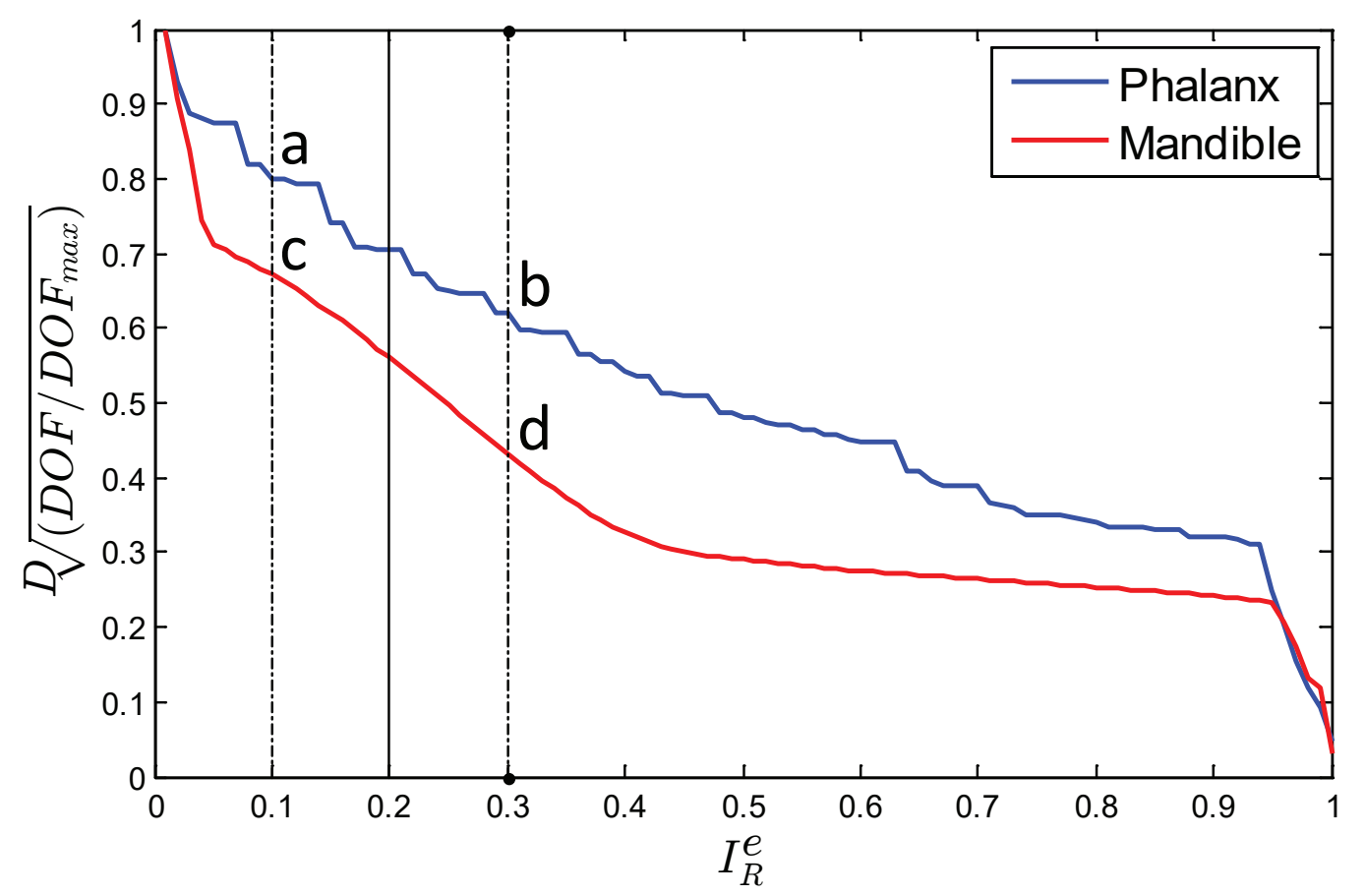

Figure 6: Mesh refinement study

of 0.2 for the $I_{R}^{e}$ index as we heuristically noticed that values around 0.2 usually lead to meshes adequate for our purpose. Figure 6 shows the mesh density index $\sqrt[D]{D O F / D O F_{\max }}$ versus the $I_{R}^{e}$ index value for the problems shown in Section 4.1 and 4.3. The mesh density index provides a relative dimension-independent estimation of the mesh density and is computed from the problem dimensionality value $D$ (which is 2 for $2 \mathrm{D}$ and 3 for $3 \mathrm{D}$ problems), the number of the degrees of freedom of the problem $D O F$ and the maximum possible value of degrees of freedom $D O F_{\max }$, which corresponds to a mesh having one element per pixel. The blue curve corresponds to the $\mathrm{X}$-ray grey scale image used in Section 3.1 whereas the red one to the CBCT scan used for the models in Section 4.2 and 4.3. In both cases the $I_{R}^{e}$ of 0.2 lays in a range of values for which the mesh refinement is sensitive to the $I_{R}^{e}$ but still easily controllable. Figures $7 \mathrm{a}, 7 \mathrm{~b}, 7 \mathrm{c}$ and $7 \mathrm{~d}$ correspond to the values of $I_{R}^{e}$ highlighted in Figure 6 as the extreme points of a range of amplitude 0.1 around the value chosen for the numerical examples.

It is worth emphasizing that no error estimation method (issue that ex- 


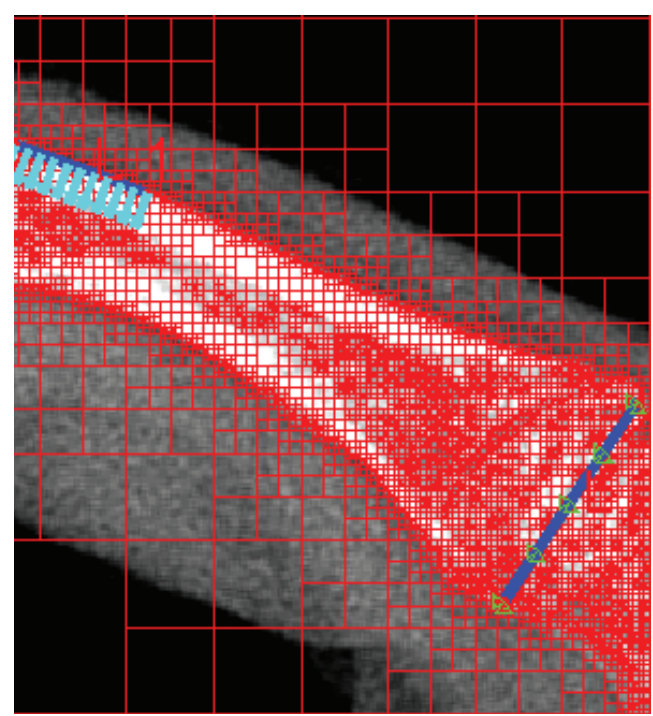

a)

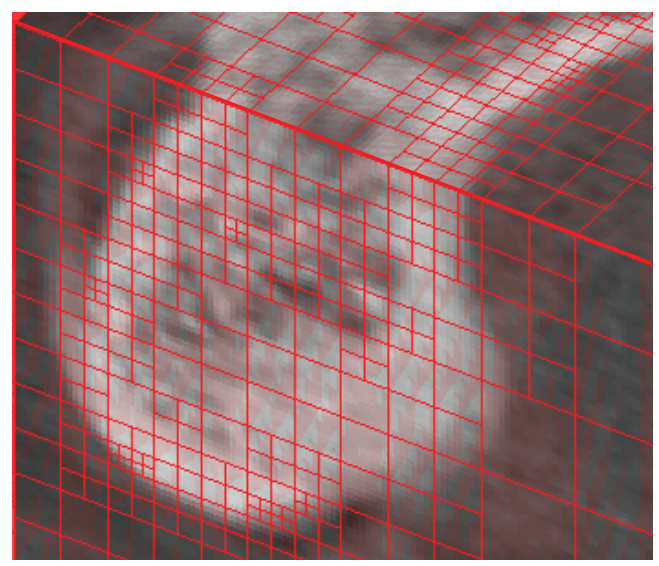

c)

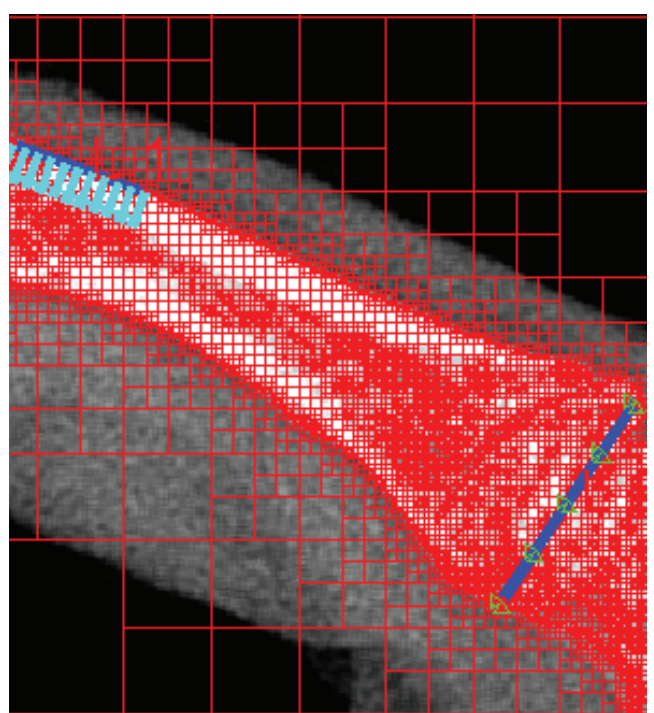

b)

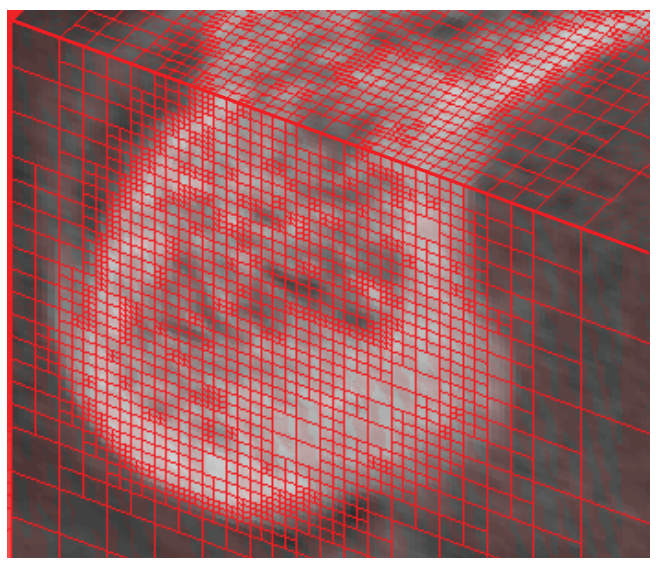

d)

Figure 7: $h$-adapted refinement sensitivity. Phalanx model of Section 4.1. a) $I_{R}^{e}=0.1$; b) $I_{R}^{e}=0.1$. Mandible model of Section 4.3. c) $I_{R}^{e}=0.1$; d) $I_{R}^{e}=0.1$.

ceeds the scopes of this paper) is proposed to guide the $h$-adaptive mesh refinement. Heterogeneity-based refinement can be considered analogous to the geometry-based one in the case of CAD-based models. As a consequence, it cannot be considered as an alternative to the use of error estimators as heterogeneity alone does not determine high stress gradient values. Nonethe- 
less, it provides accurate results with a lower number of degrees of freedom compared with uniform meshes and, in contrast to most error estimation techniques, it is inexpensive and does not require any previous calculation.

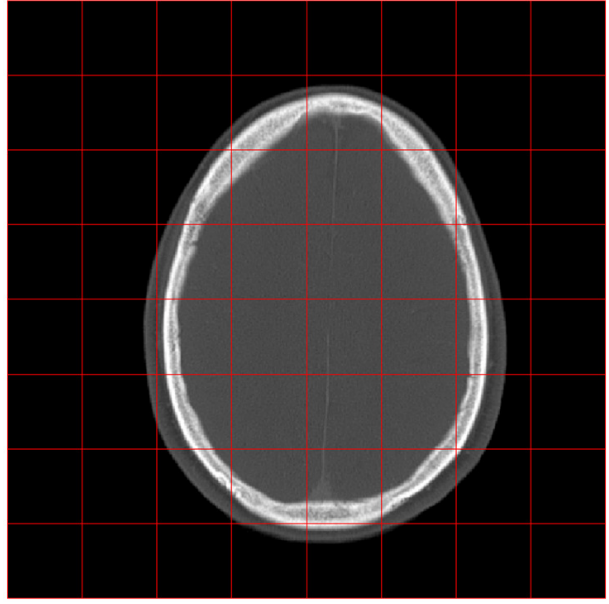

a)

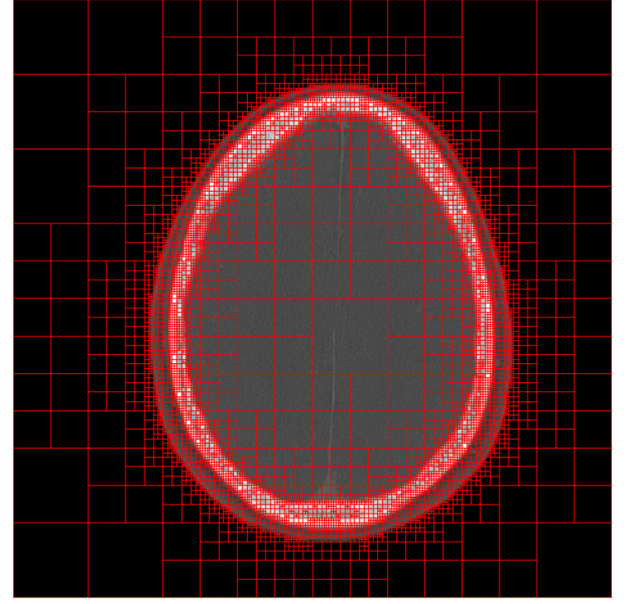

b)

Figure 8: Mesh refinement process. a) Initial uniform mesh. b) $h$-adapted mesh.

2.3. Merging image- and geometry-based cgFEM for patient specific simulation of future implants

Here we propose a procedure which takes advantage of both geometryand image-based $\mathrm{cgFEM}$ for simulating the effect of a future implant making use of a patient-specific tissue model.

The first stage of the process is reading tissue image data, see Figure 9 a), and reshaping it in order to make them suitable for the mesh $h$-adaptive process.

The prosthetic device is then introduced into the model as a closed geometry defined by a CAD representation like a NURBS (non uniform rational B-splines) surface immersed in the Cartesian grid structure which is overlapped to the bitmap, see Figure $9 \mathrm{~b}$ ).

On the one hand the pixels whose centre is contained inside the closed geometrical contour are deactivated because they correspond to the parts which have to be removed in order to insert the implant, see Figure 10 a). The pixels so ignored are the ones whose centres lay in the closed domains 


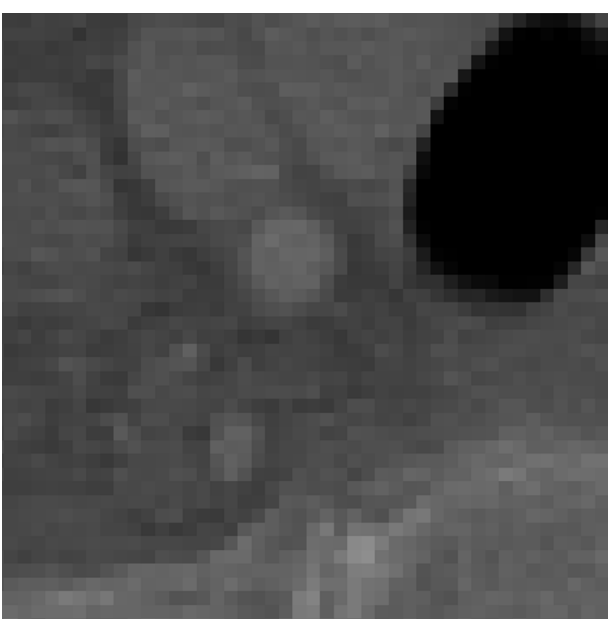

a)

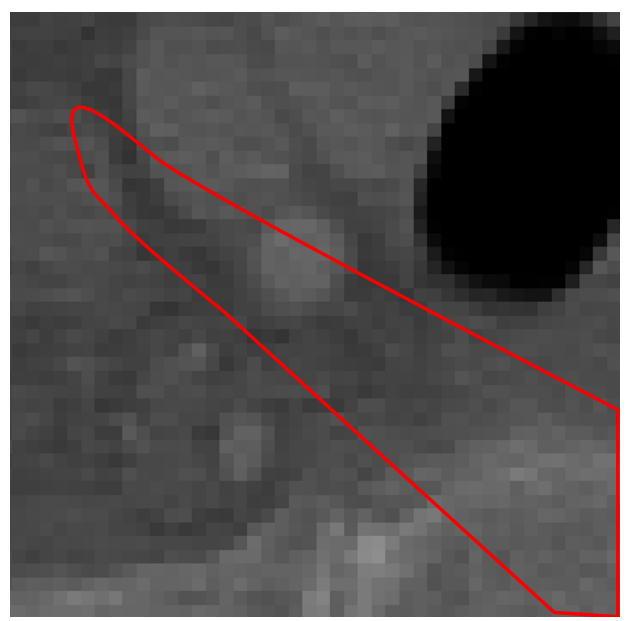

b)

Figure 9: Prosthetic device modelling. a) Bitmap domain. b) Geometric contour defined on the Bitmap domain.

of the device. On the other hand, the closed geometrical domain is assigned homogeneous material properties as in standard geometry-based $c g$ FEM, see Figure 10 a).

It is to be noted that, if the Riemann sum or the integration subdomain based integration techniques are used, the intersection between geometry and bitmap is not null neither their union perfectly covers the original image, see Figure 11 a), whereas this does not occurs in the case of least-squares fitting, see Figure $11 \mathrm{~b}$ ).

Once the mesh has been created, three categories of elements are distinguished on the basis of their position with respect to the geometrical domain. As shown in Figure $10 \mathrm{~b}$ ), the elements of the hierarchical structure can be external to the geometrical boundary (light grey elements), internal (dark grey elements) or can lie on it (white elements).

All the elements inside the same geometrical domain have proportional stiffness matrices $\mathbf{k}^{e}$, as in the geometry-based $c$ FEM, see Section 2.1, therefore the same efficient procedure is adopted. The external elements completely lie on the image, hence the same integration strategy as in image-based $c g$ FEM is adopted. The elements on the contour partially lie on both bitmap and geometry. They are divided in triangular integration sub-domains on the geometric side, as in the geometry-based $c g \mathrm{FEM}$ while, on the side of the im- 

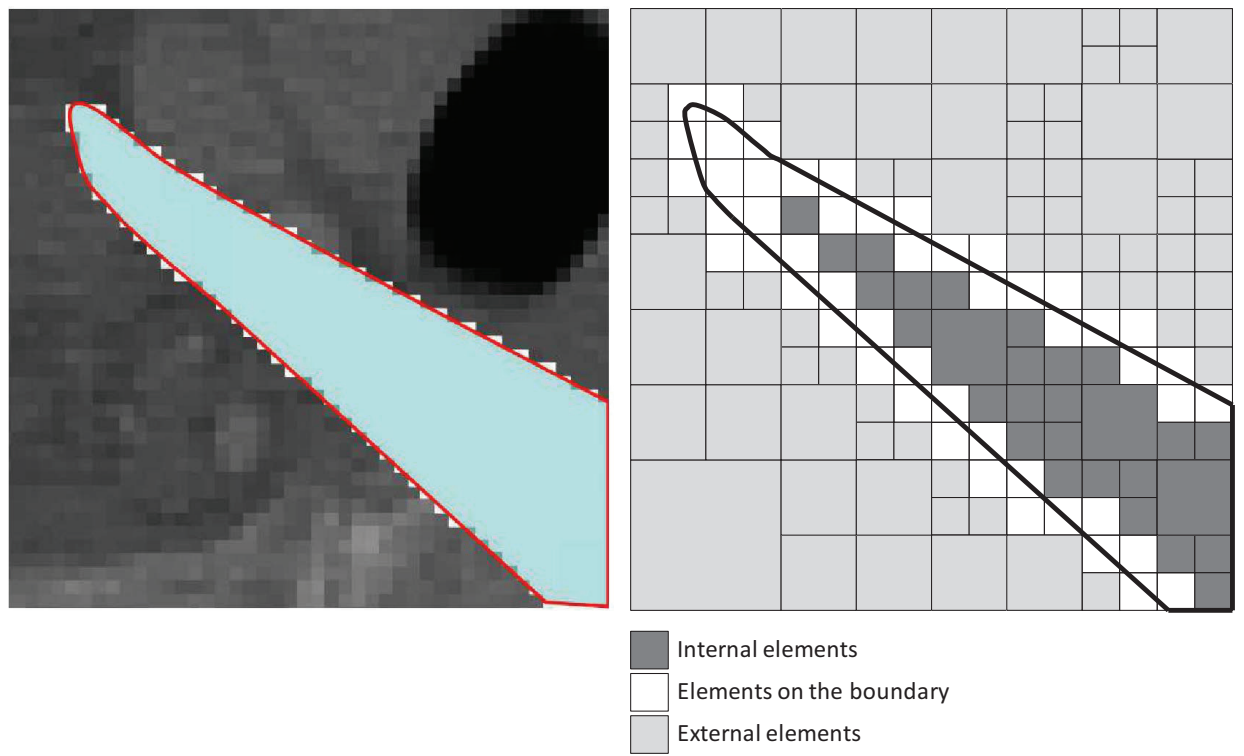

a)

b)

Figure 10: Prosthetic device simulation. a) Geometric domain and active pixels corresponding to the prosthesis in 9. b) Conceptual image of FEAVox mesh for the geometryimage mixed problem.

age, the bitmap integration presented in Section 2.2 is applied only to the active pixels, see Figure 11.

The sudden material change between prosthetic device and biological tissue is detected by the image refinement procedure, hence the mesh is refined in these areas and the inaccuracy associated to the excessive homogenization of the material properties is controlled.

\section{Validation}

In this section, we present some validation results for $2 \mathrm{D}$ models based on synthetic images focusing on the elastic property distribution and mesh size effects in Section 3.1 and on the image resolution influence in Section 3.2 .

They will show how, in $c g$ FEM, standard mesh refinement techniques such as element size reduction or interpolation order increase stop enhancing the solution at the rate expected in FEM, when the discretisation error becomes 


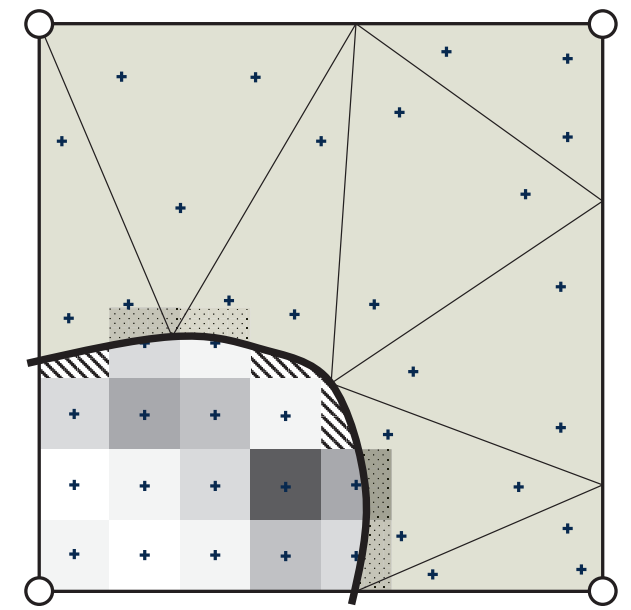

a)

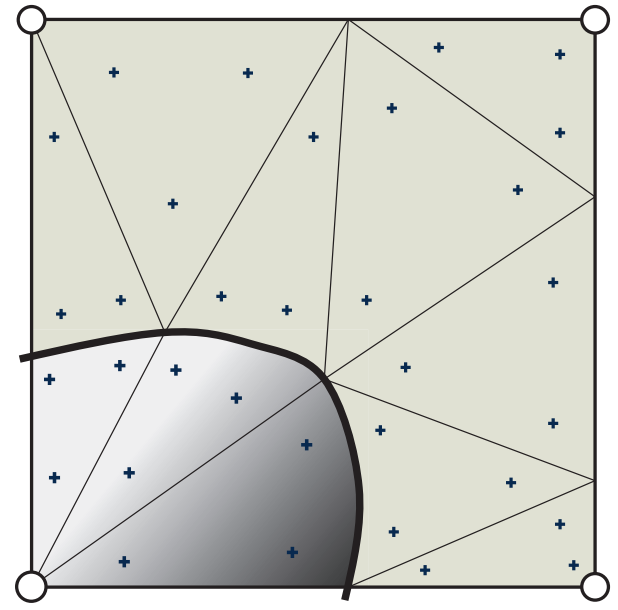

b)
Geometrical domain

Image property fitting

1 Geometric boundary $+\quad$ Integration points

Nㅔ Gap between the integration schemes

Overlapping areas

Figure 11: Representation of the integration procedure adopted on the elements on the contour of a geometrical domain in FEAVox a) Riemann Sum approach. b) Least-squares fitting approach.

smaller than the modelling error which only depends on the image resolution. As a consequence, they justify the use of linear interpolation functions and meshes coarser than the pixmap in $c g$ FEM.

As a measure of the accuracy of numerical analyses in this section we use the following error in energy norm, see Ruess et al. (2012b):

$$
e_{r}=100 \sqrt{\frac{\left|\int_{\Omega_{e x}} \boldsymbol{\sigma}^{T} \mathbf{D}_{e x}^{-1} \boldsymbol{\sigma} d \Omega-\int_{\Omega^{h}} \boldsymbol{\sigma}^{h^{T}} \mathbf{D}_{i m}^{-1} \boldsymbol{\sigma}^{h} d \Omega\right|}{\int_{\Omega_{e x}} \boldsymbol{\sigma}^{T} \mathbf{D}_{e x}^{-1} \boldsymbol{\sigma} d \Omega}}=100 \sqrt{\frac{\left|U_{e x}-U^{h}\right|}{U_{e x}}}
$$

where $\sigma^{h}, U^{h}, \Omega^{h}$ and $\mathbf{D}_{i m}$ represent the FE stress, strain energy, problem domain and material stiffness matrix respectively, whereas $\boldsymbol{\sigma}, \boldsymbol{U}_{\boldsymbol{e x}}, \boldsymbol{\Omega}_{\boldsymbol{e x}}$ and $D_{e x}$ the same magnitudes for the exact problem. The latter are calculated 
analytically if an exact solution is available as in the case of Section 3.1.1 and 3.2, otherwise they are substituted with reference values obtained from more accurate FE models, as in Section 3.1.2 and 3.1.3. Note that we have considered an absolute value in 7 to ensure a positive result that allows us to represent the evolution of this error norm in a logarithmic scale.

In order to make the integration error negligible, all the validation problems have been solved adopting the $I S$ approach to the element stiffness matrix calculation, see Equation (3). All the models in this section have a constant Poisson's ratio value of 0.3 .

\subsection{Effect of mesh size and elastic property distribution}

In this section, we study $c g$ FEM convergence behaviour in the case of uniform mesh refinement with different Young's modulus distributions for linear and quadratic FE interpolation. In order to better understand the effect of the element size, the refinement is carried out until obtaining meshes even finer than the corresponding bitmaps.

We consider three problems based on $128 \times 128$ pixel grey scale images. In the first one the bitmap is created from a geometrical model containing strong discontinuities, see Section 3.1.1, whereas in Sections 3.1.2 and 3.1.3 the images are obtained from analytical elastic property distributions.

\subsubsection{A strong discontinuity case}

The first case shows the convergence behaviour of the image-based model of a homogeneous pipe with a circular cross-section loaded by internal pressure under the hypothesis of plain strain condition according to Figure 12 a) and Table 2 . This problem is a typical benchmark in computational mechanics and has a well known analytical solution. As usual, the model is simplified by taking advantage of its symmetry, therefore, only one fourth of the pipe is studied imposing convenient symmetry boundary conditions.

\begin{tabular}{|l|l|}
\hline $\mathbf{a}[\mathbf{m}]$ & $\mathbf{3}$ \\
\hline $\mathbf{b}[\mathbf{m}]$ & $\mathbf{6}$ \\
\hline $\mathbf{P}[\mathbf{M P a}]$ & $\mathbf{1}$ \\
\hline
\end{tabular}

\begin{tabular}{|l|r|r|}
\hline Material & E $[\mathbf{M P a}]$ & Grey level \\
\hline Material 1 & $\mathbf{0}$ & $\mathbf{0}$ \\
\hline Material 2 & $\mathbf{1 0 0 0}$ & $\mathbf{2 5 5}$ \\
\hline
\end{tabular}

Table 1: Properties referred to the models in Figure 12

The image used in the simulation, see Figure $12 \mathrm{~b}$ ), was obtained by integrating a NURBs-based geometrical representation of the cylinder in a 


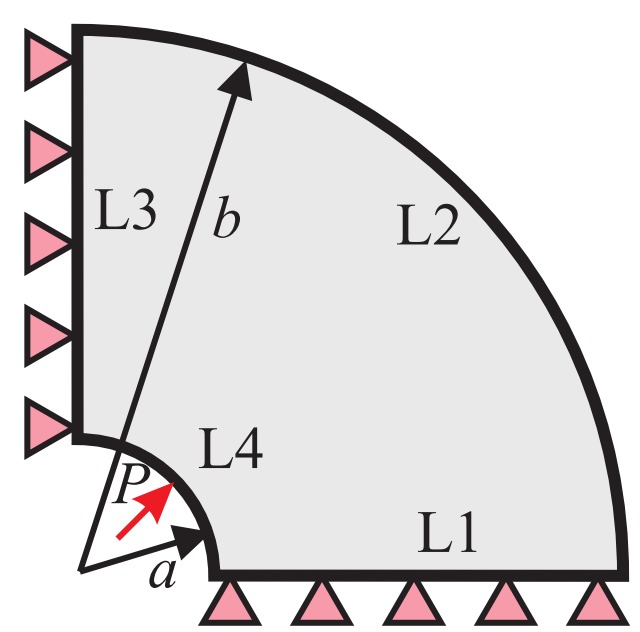

a)

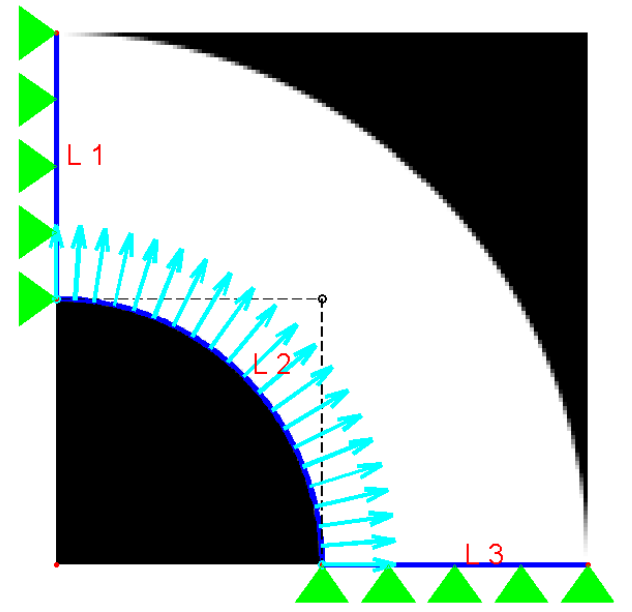

b)

Figure 12: One fourth of pipe section with internal pressure in plain strain condition, extracted from Nadal et al. (2013). a) Geometrical model. b) Corresponding bitmap model

\begin{tabular}{ll}
$\mathbf{a}[\mathbf{m}]$ & 3 \\
\hline $\mathbf{b}[\mathbf{m}]$ & 6 \\
\hline $\mathbf{P}[\mathrm{MPa}]$ & 1 \\
\hline
\end{tabular}

\begin{tabular}{|l|r|r|}
\hline Material & E [MPa] & Grey level \\
\hline Material 1 & $\mathbf{0}$ & $\mathbf{0}$ \\
\hline Material 2 & $\mathbf{1 0 0 0}$ & $\mathbf{2 5 5}$ \\
\hline
\end{tabular}

Table 2: Properties referred to the models in Figure 12

uniform mesh. This mesh had the same resolution as the desired resulting image. We evaluated the percentage area of each element covered by the cylinder and assigned a grey level proportional to this area percentage to this element. Considering each element as a pixel we created the grey scale image of the cylinder.

We chose to code the conversion from vector to bitmap representation ourselves instead of using standard graphic software. Doing so we ensured full control on the process and avoided introducing additional approximations in the final image as the procedure was intended to mimic an idealised (noise free) X-ray scan which provides grey scale values proportional to the material ratio inside the pixel area. By defining the area by a vector CAD representation, integrating it over a uniform grid and converting the pixel values into grey scale, we make sure that the only differences between the models lay in the loss of local information on the boundaries, which is pixel- 
size dependent, and in the transformation of the area values into discrete values of the grey scale.

We establish a linear relation between the Young's modulus and the grey scale computed by interpolating the values in Table 2. Doing so, we consider the Young's modulus to be proportional to the ratio of the geometrical domain contained in each pixel.

Figure 13 shows the results obtained by uniform mesh refinement of the models in Figure 12 in terms of strain energy and error. For the curves referring to the image-based model the solid trace represents super-pixel meshes, coarser than the pixmap, whereas the dash-dotted trace represents sub pixel meshes finer than the pixmap. Round markers indicates degree 1 of the interpolation functions and square ones degree 2.

The upper graph represents the ratio between the FE energy norm and the exact energy norm versus the number of degrees of freedom for image-based models with linear and quadratic interpolation functions. The horizontal black lines represent reference values: the exact solution in solid trace and an estimation for the singular pixmap solution of the image in dashed trace. This estimation was obtained by solving the image problem with a very refined quadratic mesh, corresponding to the level 9 of the nested Cartesian structure, see Figure 1. The reference lines shows the fact that the exact solution of the pixmap (which is not available) is different from the analytical solution of the geometrical model due to the piecewise discontinous nature of the pixmap. As a consequence the blue and red curves converge to this other value instead of converging to the exact solution of the geometrical problem. This is apparent for subpixel meshes, both for linear and quadratic interpolations. Due to the fact that, in this case, the estimation for the image singular solution is higher than the exact one, the image-based convergence curves intersect the solid black line, for a lower number of degrees of freedom in the case of quadratic interpolation functions. These intersections are marked by vertical dotted lines which are prolonged to the lower graph because they are relevant to understand the error behaviour.

In the lower graph the results are plotted as relative error in energy norm versus degrees of freedom. As a reference, we plot the geometrical model error convergence curves calculated with the geometry-based $c g$ FEM technique. They are black lines characterised by the theoretical slope values of 0.5 and 1 for linear and quadratic interpolation functions.

As in the upper graph, the blue and red curves refer to image-based models with linear and quadratic interpolation functions. Error is calculated 

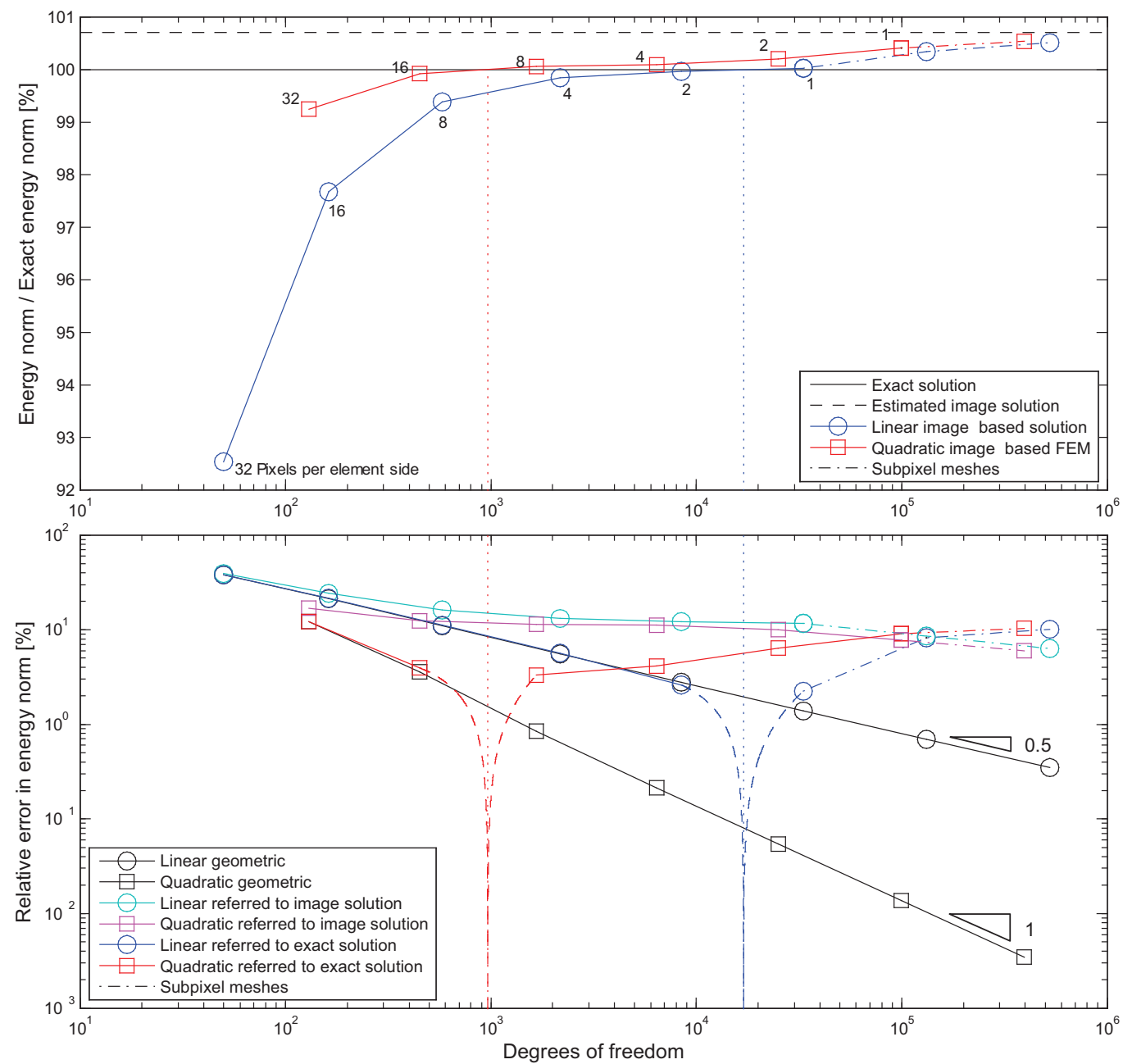

Figure 13: Relative energy norm and error convergence of the problem shown in figure 12 with uniform mesh refinement and image resolution of $128 \times 128$ pixels

using the analytical strain energy value of the reference geometrical problem in Equation (7). As a consequence, in each of them an ideal point can be individuated which corresponds to the relative intersection in the upper graph and for which the error equals 0 . The vertical dotted lines are, therefore, asymptotes for the error curves and the asymptotic parts of the blue and red curves are represented with a dashed trace.

These 0 error points do not correspond to any actual mesh and will only 
appear if the singular image solution has a strain energy higher than the exact solution. They are the result of a strain energy compensation between the discretisation and the modelling error since the former decreases throughout the refinement process, as in usual FEM, whereas the latter increases because finer meshes better capture the difference between the pixmap and the geometrical model.

As the number of degrees of freedom increases beyond the vertical asymptotes, the blue and red error curves significantly diverge from the respective geometrical FE solutions. As a consequence, they lose the theoretical convergence rates in logarithmic scale of 0.5 for linear and 1 for quadratic interpolation functions.

For linear interpolation functions, the geometry and image-based solutions behave alike provided that the elements are bigger than the pixels and the solution clearly diverges for sub-pixel meshes (represented as dash-dot lines). In the case of quadratic elements, in contrast, this phenomenon starts appearing for coarser meshes because of the higher discretisation error reduction rate.

In the lower part of Figure 13 we also plot the image-based model error convergence curves with respect to the image solution, in cyan and magenta for linear and quadratic elements respectively. They result from substituting the exact strain energy $\boldsymbol{U}$ in Equation (7) with the estimation of the pixelised model solution corresponding to the black dashed line in the upper graph.

As the mesh is refined these curves tend to have the same slope. This behaviour is reasonable considering that the pixelised model is piecewise discontinuous and, as a consequence, the singularities prevent the convergence from reaching the theoretical values guaranteed for problems with smooth solutions. The convergence slope is, therefore, determined by the singularities and is independent from the FE interpolation order.

In this numerical example, the bitmap intrinsic inaccuracy in the domain boundary representation prevents the numerical solution from converging to the exact one with the increase of the number of degrees of freedom. In contrast a limit appears for which the increase of computational cost due to the mesh refinement ceases to reduce the error with respect to the analytical solution. In this case this limit can be set in a neighbourhood of $\mathbf{2} \times \mathbf{2}$ and $16 \times 16$ pixels per element for linear and quadratic FE interpolation respectively. Refining the mesh beyond these values is uneconomical. The number of mesh levels suitable for the simulation is broader for linear FE interpolation functions, as a consequence, this polynomial order appears more 
suitable for the $h$-refinement of $c g$ FEM.

\subsubsection{A smooth Young's modulus distribution}

Figure 15 shows the relative energy norm and error versus the degrees of freedom for the plate $[-0.5,0.5] \times[-0.5,0.5]$ loaded with uniform unit tension on the upper side and symmetry boundary conditions on the lower and left sides. The Young's modulus distribution is expressed in Equation (8) and shown in Figure 14 a) with the problem boundary conditions. The $128 \times$ 128 pixel image was obtained computing the analytical mean value of the Young's modulus in the area of each pixel and converting it into grey scale.

$$
E(x, y)=(y+10)(x+10)^{2}
$$

In the image-based models we establish a linear relation between colour and

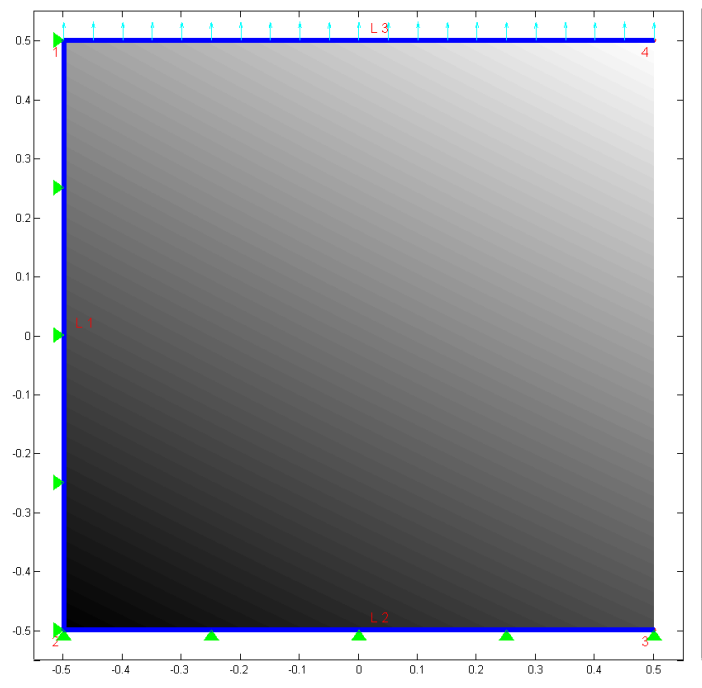

a)

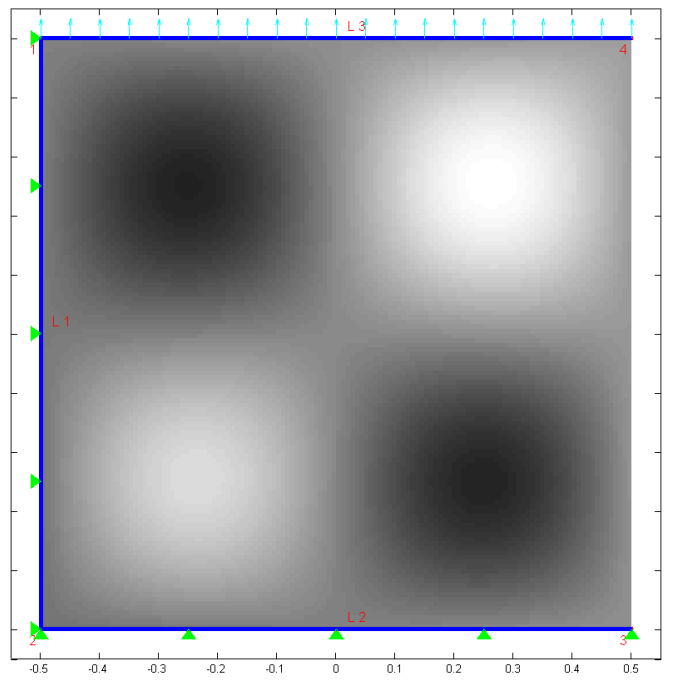

b)

Figure 14: Square plate models representing the smooth variations of Young's modulus given in Equation (8) and 9 respectively

stiffness in such a way that the minimum of the function in Equation (8) on the problem domain corresponds to 0 and its maximum to 255 in grey scale.

In Figure 15, as in the previous section, the upper graph compares the energy norm convergence of the image-based models for linear (blue) and quadratic (red) shape functions. The reference and the estimated image 

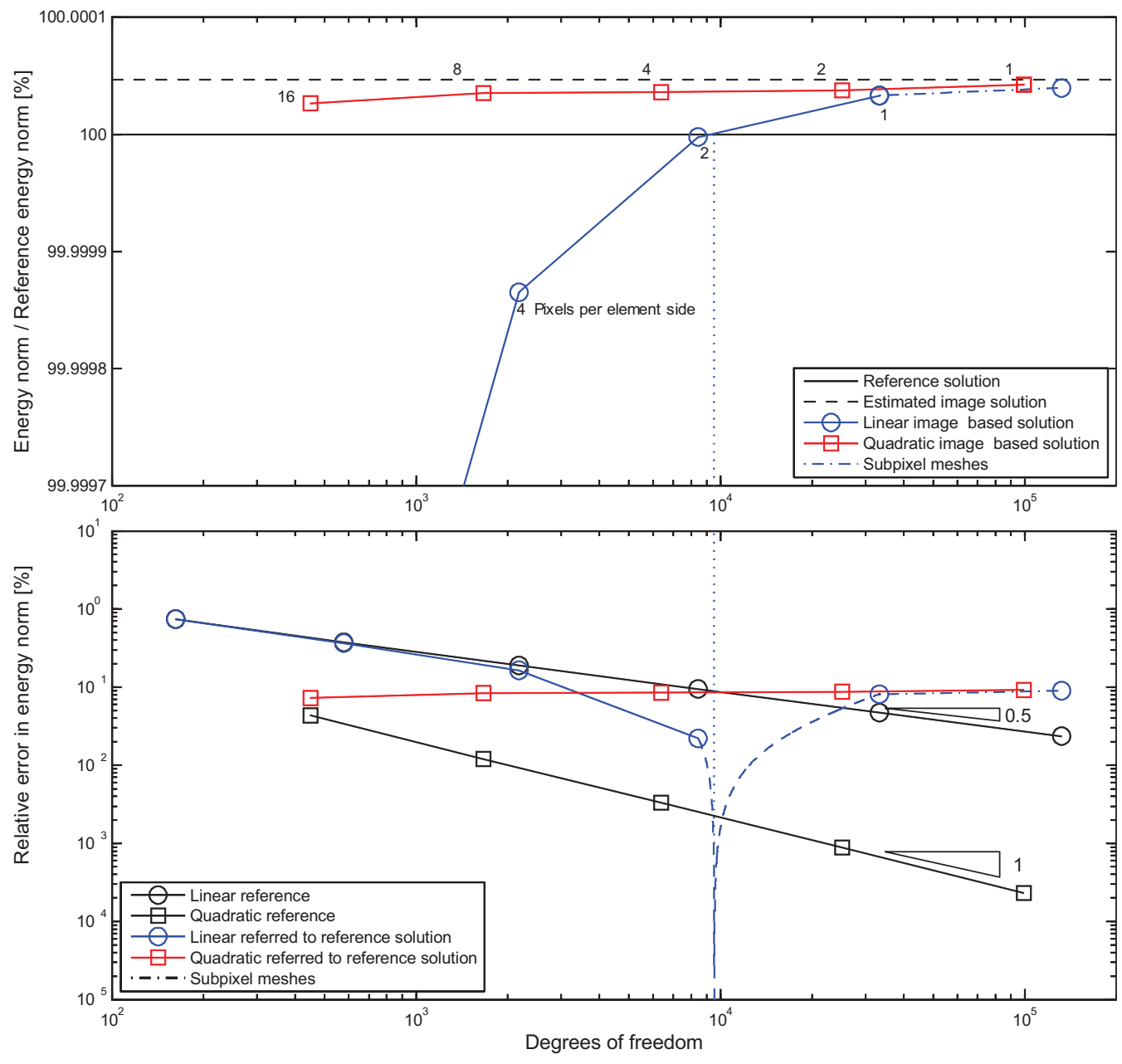

Figure 15: Relative energy norm and error convergence of the problem shown in figure 14 a) with uniform mesh refinement and image resolution of $128 \times 128$ pixels

solutions are traced as solid and dashed black lines respectively. These solutions were computed with quadratic 8 level meshes integrating the element stiffness matrix with the analytical Young's modulus distribution in the first case and with the value from the $128 \times 128$ bitmap in the second one. As in the problem of Section 3.1.1, the estimated strain energy is higher for the discontinuous model than the reference one and, as a consequence, the blue and red curves intersect the solid black line. The intersection can actually be seen only in the case of linear interpolation functions in Figure 15 for an 
element size slightly lower than $2 \times 2$ pixels per element. Note that, in this case, the convergence curve starts diverging from the theoretical behaviour of the continuous model for a coarser mesh than in the previous numerical example. In the quadratic case is occurs for a coarser mesh outside the represented range. The intersection is marked and prolonged to the error graph and used to draw the asymptotic curve branches. In the lower graphs the reference problem black curves appear to converge according to the theoretical slope values for linear and quadratic interpolation functions. Despite the fact that in this case the reference and the estimated image solution are closer to each other, the behaviour of the image-based simulation error is similar to the one in Section 3.1.1 for linear interpolation functions (blue line). In contrast, for the quadratic meshes (red line), the discretisation error is negligible compared with the modelling one. As a consequence, the corresponding error in the lower graph does not follow the reference problem but directly converges to the modelling error. Note that, for linear interpolation functions, the error convergence curve is less stable than in the previous cases and shows a certain divergence from the theoretical curve even at $\mathbf{2} \times \mathbf{2}$ pixels per element.

\subsubsection{A bisinusoidal Young's modulus distribution}

We obtained the model in figure 14 b) by substituting the Young's modulus distribution in Equation (8) with the one in Equation (9) for the plate in Section 3.1.2.

$$
E(x, y)=(y+10)(x+10)^{2}(1+0.7 \sin 2 \pi x \sin 2 \pi y)
$$

The results, shown in Figure 16, are computed as in the previous problem. In contrast to the model in Section 3.1.2, in this case the energy norm value of the reference solution is higher than that of the estimated image solution ergo neither the blue nor the red curve intersects the exact solution, therefore the asymptotic part is missing in the error curves of the second graph.

Since there is no difference between the way the models shown in Figure 14 are obtained, we deduce that the mutual relation between the exact strain energies of the continuous model and discontinuous pixelised one is problem dependent.

Similarly to the results in Figures 13 and 15, the error behaviour in the linear interpolation case in Figure 16 follows the reference problem for meshes coarser than the pixmap whereas the mesh refinement stops reducing the error value for a very low number of degrees of freedom for quadratic elements. 

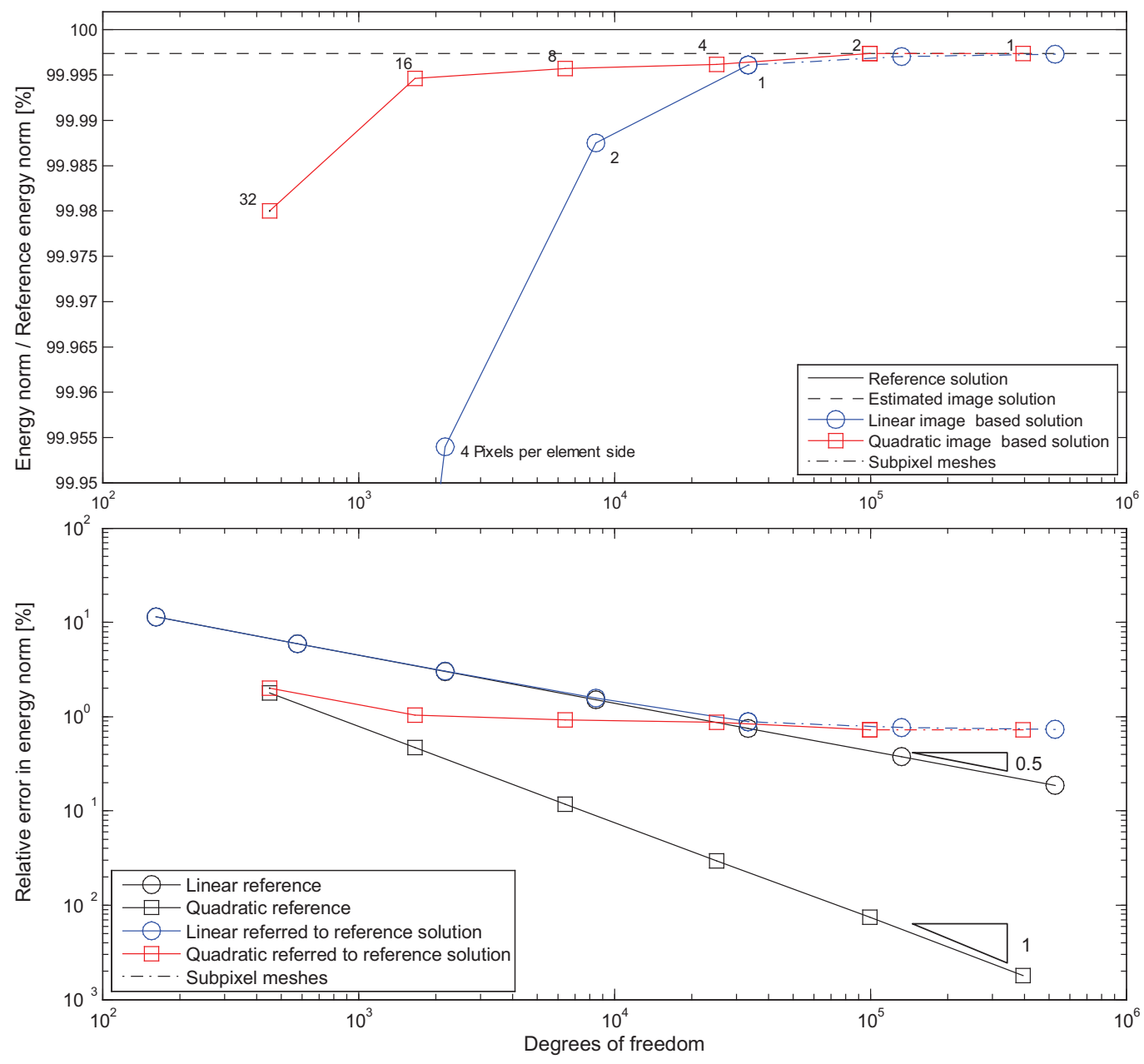

Figure 16: Relative energy norm and error convergence of the problem shown in figure 14 b) with uniform mesh refinement and image resolution of $128 \times 128$ pixels

As in the previews case, the cause is an intrinsic bitmap model error which cannot be reduced by the spatial or order refinement of the mesh. In contrast to the model in section 3.1, in this case the model error is related to the discontinuous elastic property representation instead of the boundary inaccurate definition. 


\subsection{Image resolution effect}

In order to evaluate the influence of image resolution on $c g$ FEM results, we study the behaviour of numerical models based on images of $128 \times 128$, $256 \times 256,512 \times 512$ and $1024 \times 1024$ pixels obtained from the geometrical model shown in Figure 12 a) following the same procedure described in Section 3.1.1.

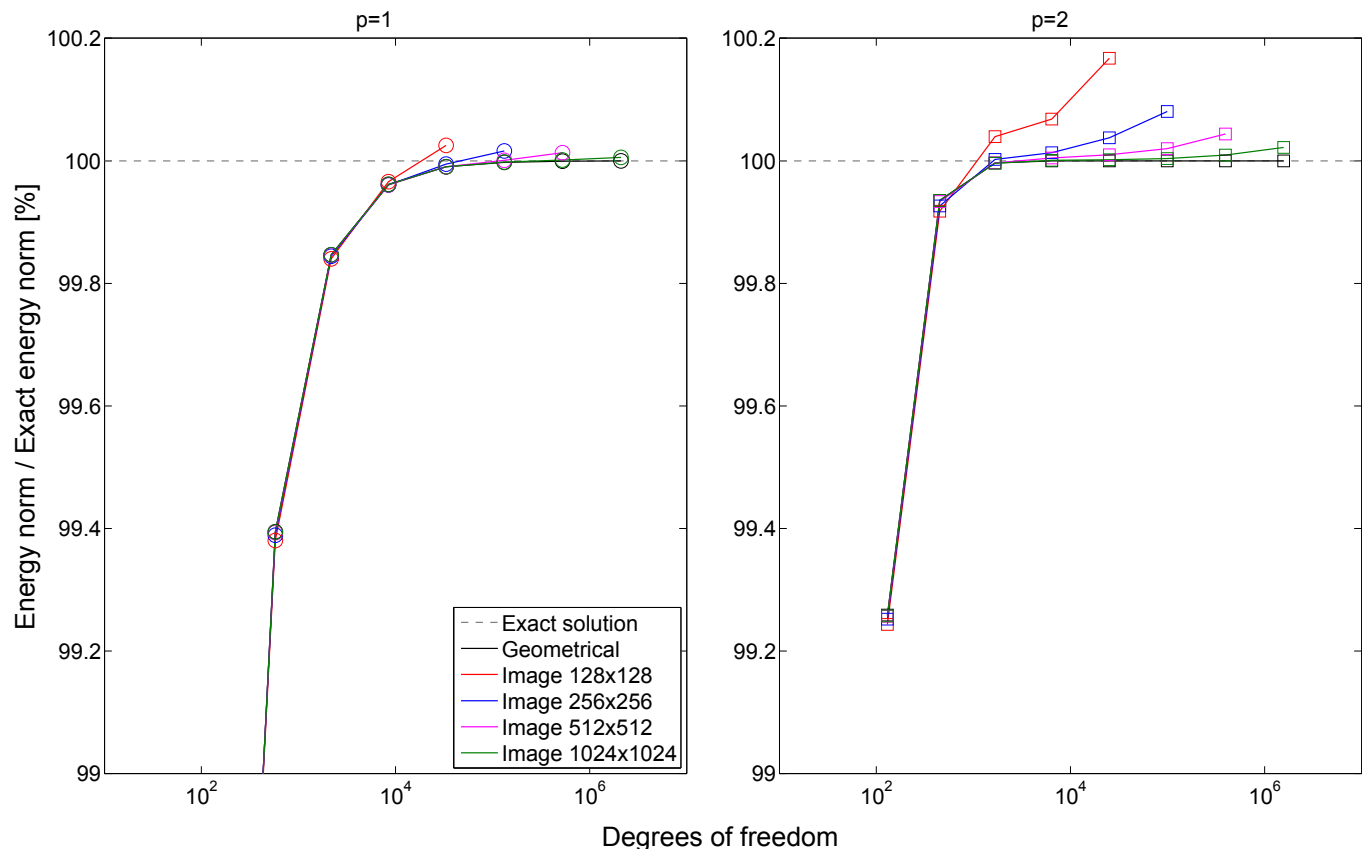

Figure 17: Relative energy norm for the model in figure 12 with different resolutions and uniform refinement

The convergence curves for linear $(\mathrm{p}=1)$ and quadratic $(\mathrm{p}=2)$ interpolation functions are shown in terms of relative strain energy versus degrees of freedom in the same scale in Figure 17. In this case we only show results for super-pixel meshes with a minimum number of 1 pixel per element, that is, no sub-pixel mesh was used, in contrast to Section 3.1.1. As a reference the exact solution is shown as a dashed black line. As to be expected, in both graph the higher the image resolution, the closer the solution strain energy is to the exact one. As in Figure 13, all the curves intersect the exact solution and tend to higher strain energy values, which tend to get closer to the exact value as the image resolution increases. Nevertheless, in the range of 

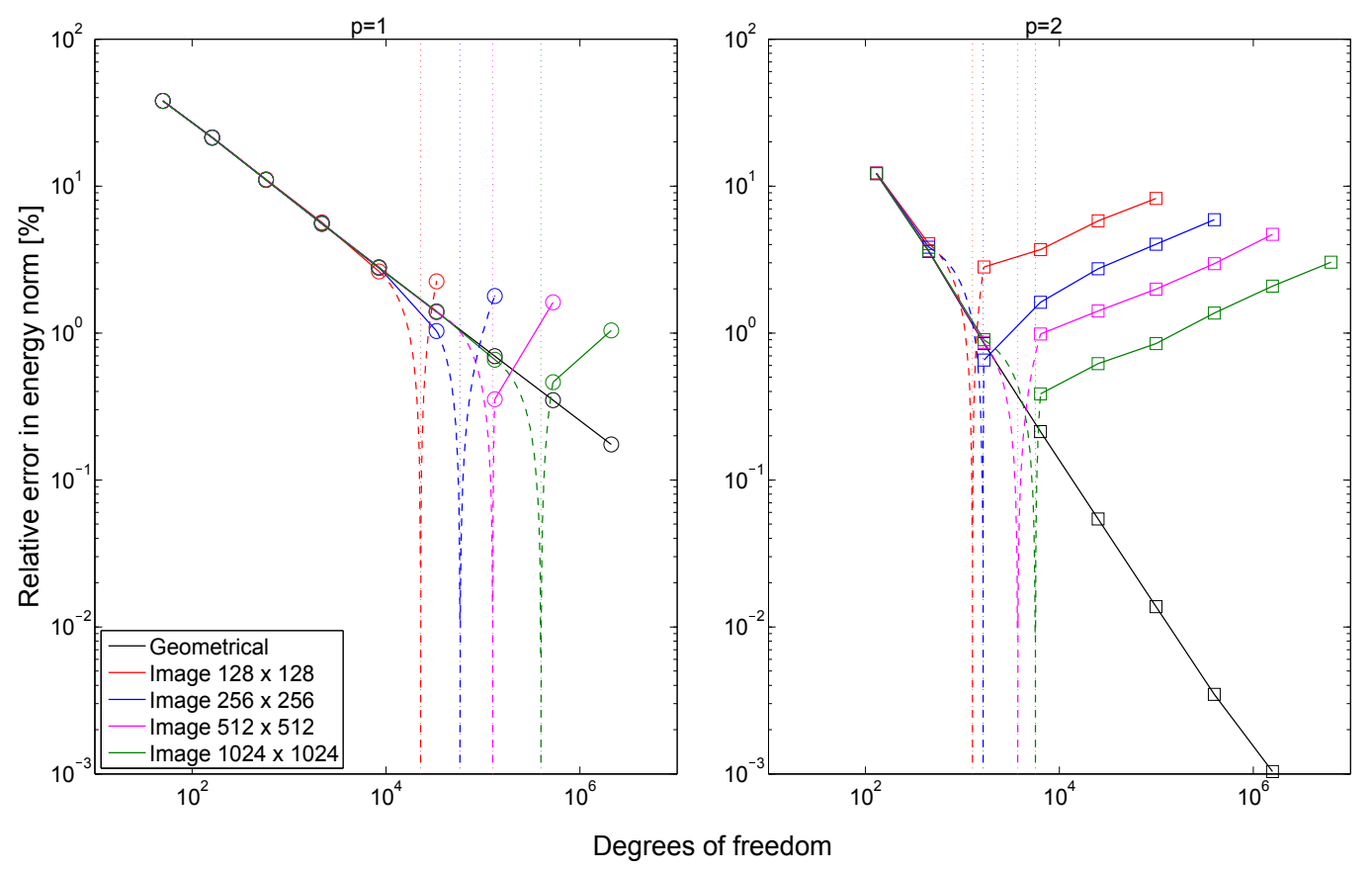

Figure 18: Relative error in energy norm for the model in figure 12 with different resolutions and uniform refinement

super-pixel meshes, the use of linear shape function has a smother approach to the exact solution.

This can also be seen in Figure 18, in which the relative error in energy norm versus degrees of freedom is plotted. As in Section 3.1.1, the error was calculated according to Equation (7) using the benchmark problem exact solution. In this case, the black curves represent the geometry-cgFEM convergence curves with the theoretical slopes of 0.5 for linear and 1 for quadratic interpolation functions. The asymptotic part of the curves tends to appear earlier with respect to the most refined mesh, as the image resolution increases. Nevertheless in the case of linear interpolation functions, the error curves closely follow the geometrical results if the refinement is not carried out beyond the element size corresponding to $2 \times 2$ pixels per elementor $4 \times 4$ pixels in the case of the $256 \times 256$ pixels image. For quadratic interpolation functions, the curves lose the theoretical slope for coarser meshes.

WithTthese and the previous results we can conclude that, in general, linear elements guarantee a reasonable convergence rate as long as the elements contain a number of pixels higher than what we can call the reliability 
limit that would be $2 \times 2$ or $4 \times 4$ pixels, depending on the problem and the image resolution. This implies that, if we consider our target solution as the exact continuous one, which in the general case is unknown and cannot be estimated by FE refinement, it may be convenient to carry out simulations with super-pixel meshes and that in traditional pixel-based methods the increase of computational cost does not correspond to the accuracy enhancement expected in geometry-based FE. In addition the availability of a higher number of valid mesh levels for which the theoretical convergence rate is kept makes linear interpolation function more suitable for $h$-refinement.

On the other hand, if we consider our target the singular solution of the image-based problem, as soon as the mesh refinement starts capturing the problem singularity, this determines the convergence rate of the solution independently from the order of the FE interpolation functions.

Taking into account these considerations, all the problems presented from now on are solved with linear elements.

\section{Numerical results}

The following are numerical results based on real medical images and obtained by using linear interpolation functions and $h$-adapted meshes.

In Section 4.1 we use a 2D X-ray scan to study the performance of different integration techniques comparing uniform and colour-based $h$-adapted meshes.

In Section 4.3 we show an application in 3D for which the model is obtained from the CT scan of a human jaw.

Finally, in Section 4.2 , we present the 3D model of a dental implant as an example of interaction between the the geometry and image-based $c g$ FEM techniques.

\section{1. cgFEM application to 2D X-ray scan. Quadrature rule influence}

In this section we evaluate the behaviour of the different integration procedures explained in Section 2.2 with uniform and colour-coded $h$-adapted refinement on a real X-ray scan. For this purpose, we use the phalanx 2D model in Figure 19.

The relation between Young's modulus and image grey levels is considered as a $\boldsymbol{C}_{\mathbf{0}}$ continuous, piece-wise lineal interpolation of the values in Table 3 extracted from the literature, see Viceconti et al. (1998) and Kim et al. (2010). Regarding the Poisson's ratio, we used a uniform value of 0.3. 


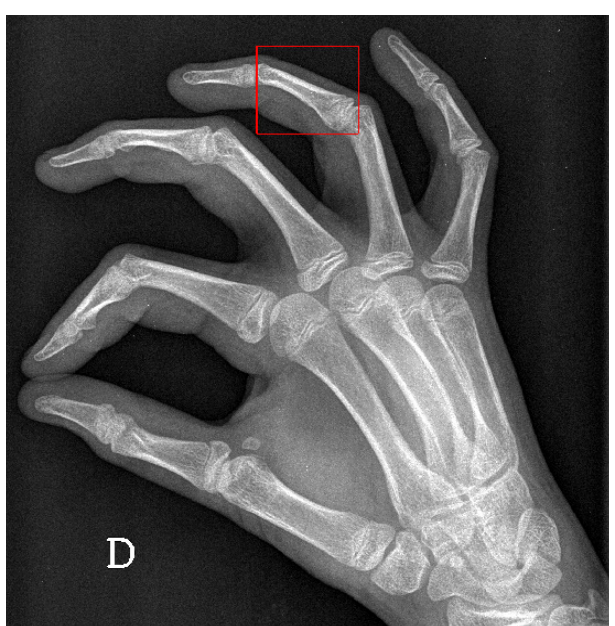

a)

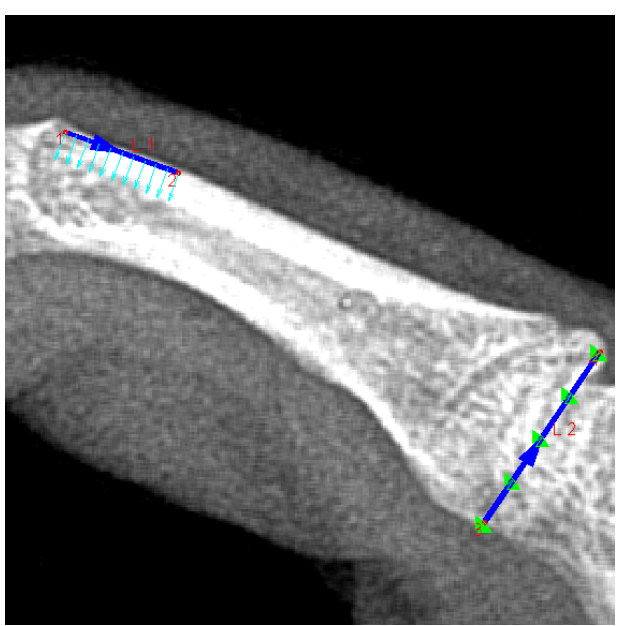

b)

Figure 19: a) Hand X-ray image. b) Phalanx image used in the simulation. The curves used for the boundary condition imposition are shown.

\begin{tabular}{|l|l|l|l|l|}
\hline Material & Air & Muscle & Bone 1 & Bone 2 \\
\hline Grey level & $\mathbf{0}$ & $\mathbf{1 5 0}$ & $\mathbf{1 9 0}$ & $\mathbf{2 5 5}$ \\
\hline E [GPa] & $\mathbf{0 . 0 0 0}$ & $\mathbf{0 . 6 4 5}$ & $\mathbf{1 4 . 0 0 0}$ & $\mathbf{1 4 . 2 0 0}$ \\
\hline
\end{tabular}

Table 3: Material Properties referred to Figure 19.

The area of interest of the X-ray image was selected, see 20 a), and two curves were created in order to apply a null displacement Dirichlet boundary condition, represented with green triangles, and a $1 \mathrm{MPa}$ uniform pressure Neumann boundary condition, represented with cyan arrows, see Figure 19 b).

Figure 20 shows the meshing step and the von Mises stress distribution for an $h$-adaptive refinement on a coarse uniform mesh of level 4 up to level 8 of the Cartesian structure, limiting the maximum value of the variation index of the pixel field to be $\boldsymbol{I}_{\boldsymbol{R}}^{\boldsymbol{e}} \leq 0.2$, see Equation (6). In each refinement step the elements which do not satisfy this condition are split into 4 new elements. The new element neighbours are also refined, in turn, to guarantee the maximum level difference between adjacent elements is not greater than 1. Note that although the different biological tissues have not been explicitly segmented, the mesh refinement process automatically identifies the bound- 


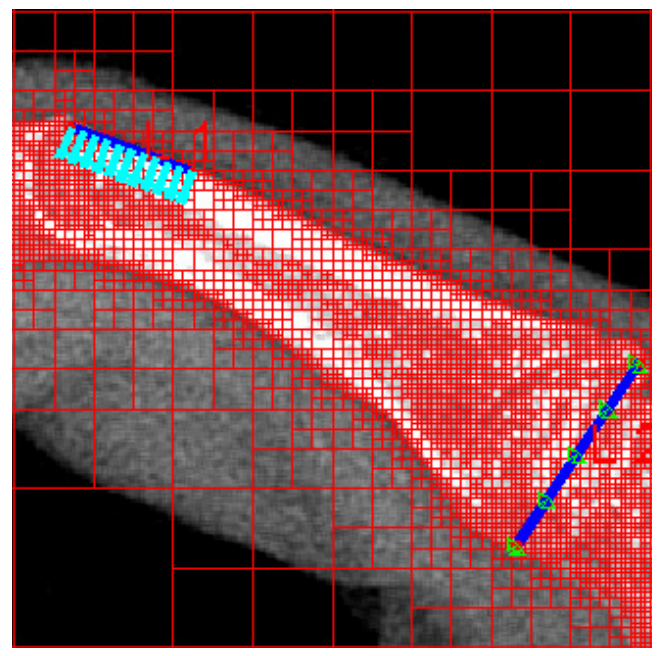

a)

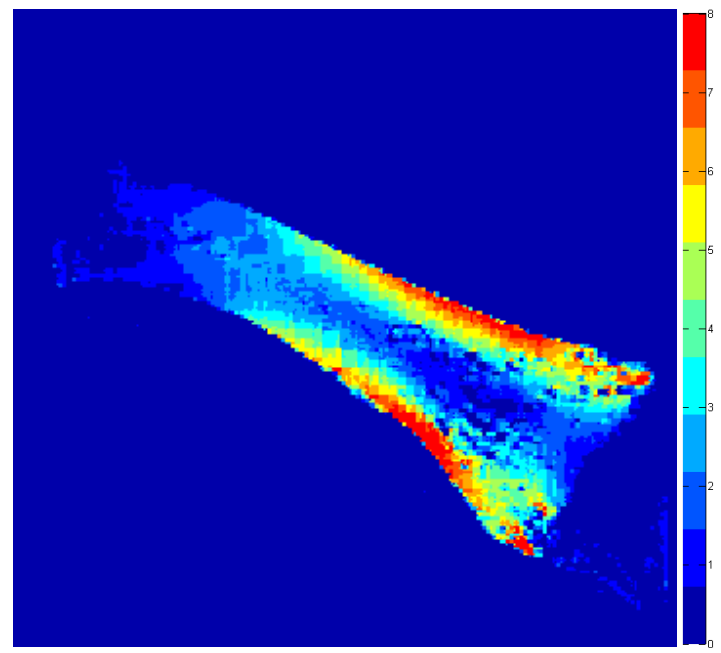

b)

Figure 20: a) $c g$ FEM mesh obtained by range evaluation guided $h$-adaptivity. b) von Mises stress distribution

aries of the tissues and refines the mesh to properly capture their geometry. The von Mises stress field calculated at the integration points is shown in Figure $20 \mathrm{~b}$ ). It is apparently consistent with the problem characteristics even though in 2D X-rays the effect of the projection along the third dimension makes it difficult to distinguish between different materials.

The convergence study in Figure 21, carried out with uniform refinement, shows the performance of the different quadrature described in Section 2.2. The problem strain energy is plotted versus the number of degrees of freedom (DOF), in the first chart, and versus the mesh total number of integration points (IP), in the second chart. Both of them are relevant because, on the one hand, the number of degrees of freedom affects the time and hardware requirements necessary to solve the $\mathrm{FE}$ system of equations and, on the other hand, a high number of integration points increases the computational resources necessary to create the aforementioned system of equations.

In Figure 22 we compare the behaviour of uniform (dashed) and $h$ adapted (solid) meshes in terms of strain energy versus degrees of freedom.

In both figures, the blue curves refer to the integration by subdomain decomposition, see Equation (3), the red ones to the Riemann sum based integration, see Equation (2), and the magenta curves to the integration 


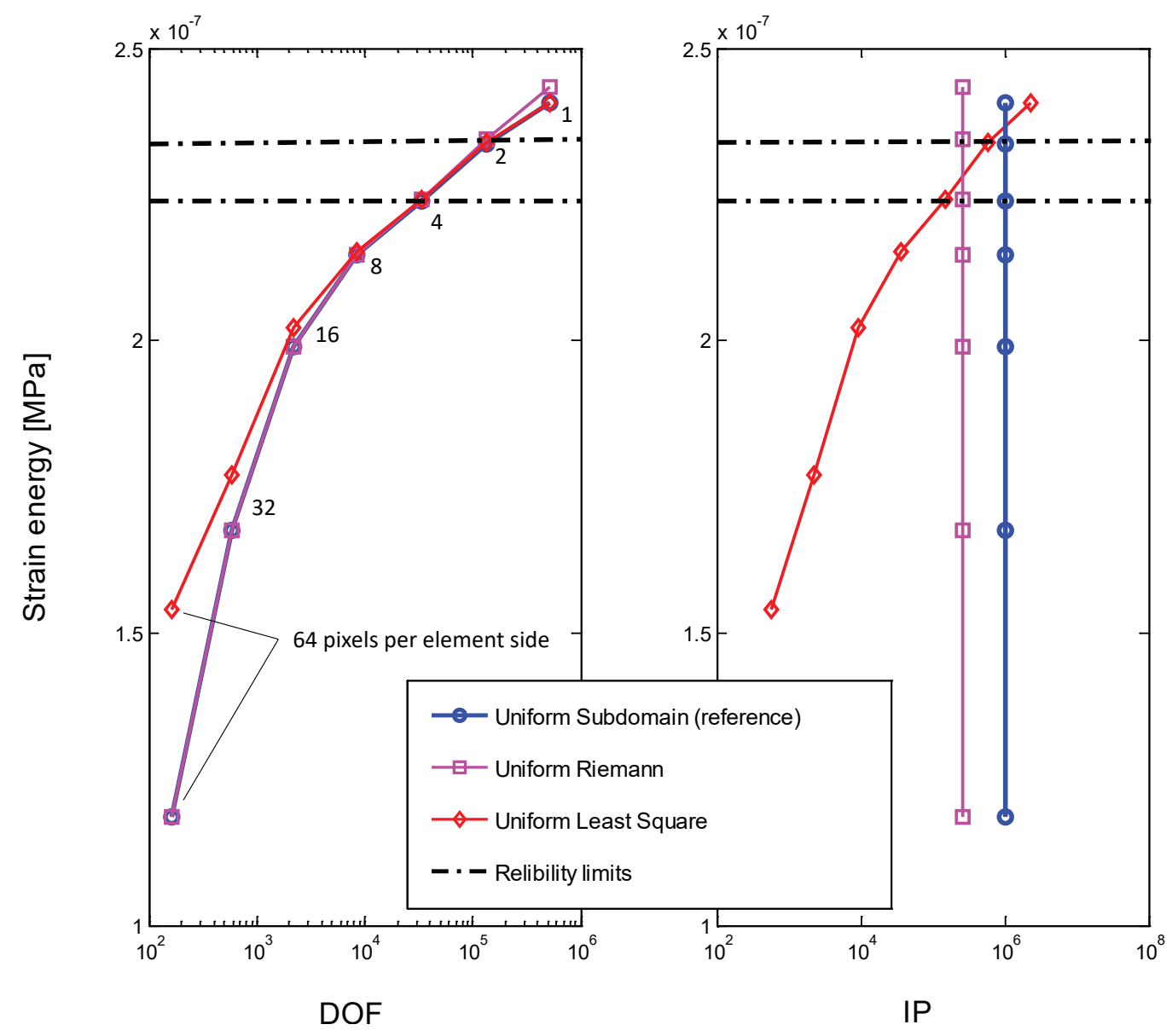

Figure 21: Strain energy versus number of degrees of freedom (left) and integration points (right) for uniform refinement of the model in Figure 21 for different integration schemes.

based on least square fitting, see Equations (4) and (5).

The integration by subdomain decomposition is the one used in the validation results, see Section 3. In general, it is very expensive because the model total number of integration points is high and independent from the mesh. Nonetheless, it does not introduce any relevant further error source since it implies the use of the adequate Gauss quadrature and the direct use of the image information. As a consequence, we can adopt it as a reference to judge the performance of the other integration techniques.

All the results in Section 3 show that, for linear shape functions, the difference between the convergence curves of the geometry-based solution 


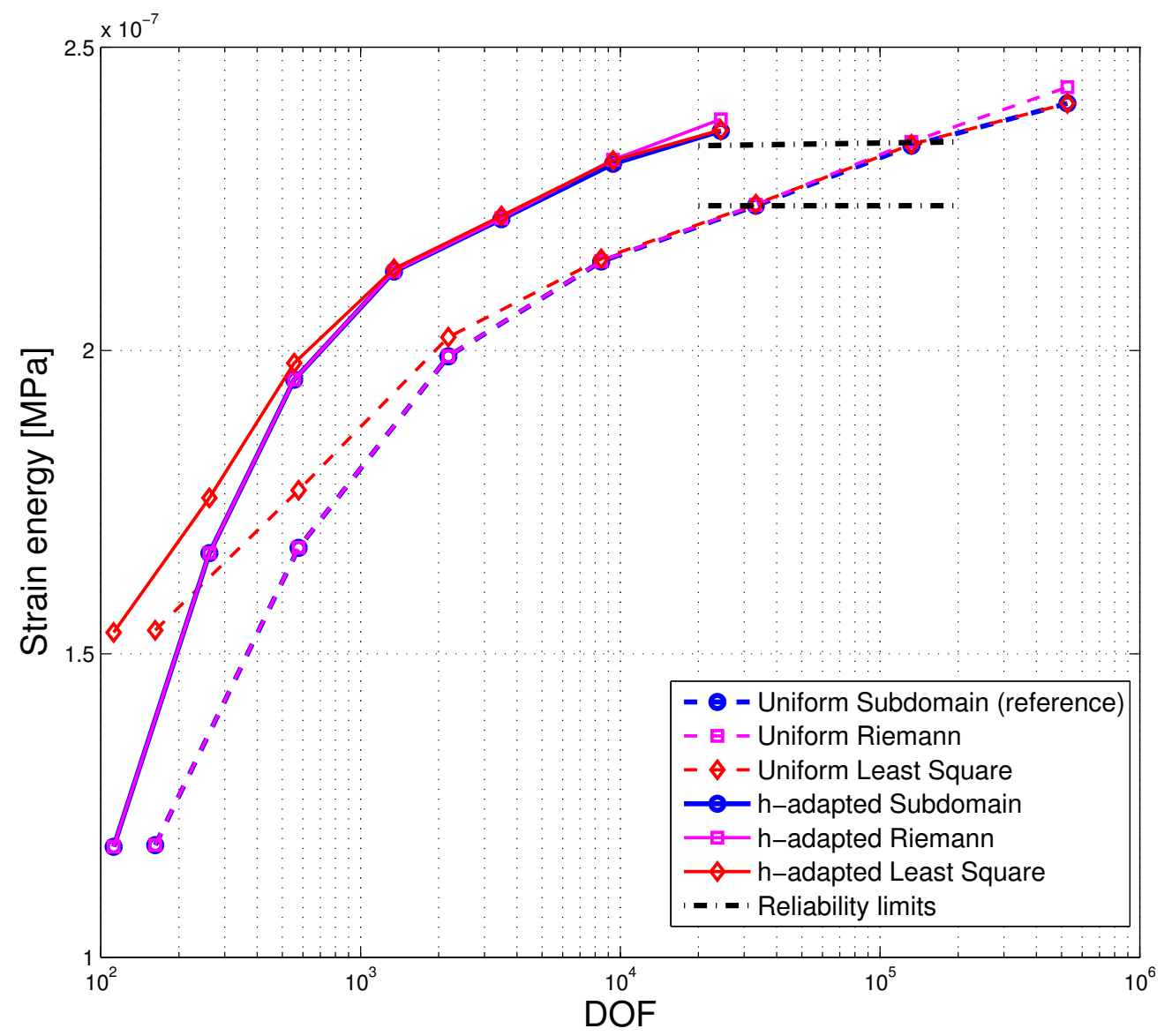

Figure 22: Strain energy versus number of degrees of freedom for uniform and $h$-adapted refinement of the model in Figure 21 for different integration schemes

and the image-based image-based ones is negligible provided that at least $4 \times 4$ pixels are contained in each element. For such an element size the pixelised nature of the model shows to have a very small influence on the results. On the basis of this consideration, we plot the black dash-dotted horizontal line which corresponds to the strain energy associated to this mesh size as a reliability limit for the mesh refinement.

In Figures 21 and 22, the red curve appears to be as accurate as the blue one for values below the reliability limit, nonetheless it is less expensive because it only has one-fourth the amount of integration points. For finer meshes, beyond the dash-dotted line, it results in a reduced integra- 
tion scheme that can influence the accuracy of the element stiffness matrix $\boldsymbol{k}_{\boldsymbol{e}}$. On the contrary the least square-based integration scheme, magenta curve, underestimates the element stiffness for coarse meshes. In contrast to the other two integration techniques, with the least square-based integration the number of integration points depends on the mesh refinement. For coarse meshes the number of integration points is lower whereas for very fine meshes, beyond the reliability limit, it becomes slightly higher than the subdomain decomposition and Riemann sum-based techniques.

Figure 22, shows how the integration technique behaviour does not change in the case of $h$-adapted refinement and how $h$-adapted meshes can provide similar strain energy values as the uniform ones with fewer degrees of freedom and, as a consequence, with a lower computational cost. The uniform refinement test shows that the LS technique appears as accurate as the reference integration scheme for meshes consisting of elements which contain less than $16 \times 16$ pixels. For larger elements the LS elastic property smoothing appears to underestimate the element stiffness, which, in this particular case, makes the global energy of the solution closer to the convergence value than the other integration techniques. LS provides fewer IP in the mesh than the Riemann sum for elements containing more than $2 \times 2$ pixels. In contrast, figure 22 shows that, if a proper number of refinement levels is available, the $h$-refinement process detects and splits the large heterogeneous elements unsuitable for the LS integration and provides accurate, less expensive results. On the basis of these results and the aspects highlighted in section 2.2, we consider the LS integration technique an effective way to reduce the computational cost in the context of heterogeneity-based $h$-adaptivity.

\subsection{A 3D cgFEM jaw bone model}

As a mere example of efficiency of the meshing process, in Figure 23 we show one eighth of an $h$-adapted model obtained from a cone beam CT (CBCT) without previous segmentation. This consists of about 2.8 million nodes and was obtained in 63 seconds with an Intel(R) Core(TM) i7-3770K $3.50 \mathrm{GHz}$ and a RAM of $16 \mathrm{~GB}$ of $451 \times 451 \times 451$ voxels. It is not suitable for calculation because of the impossibility of assigning univocal elastic properties to the pixel values. For this reason, we reduced the analysis area to the sole bone by segmenting the CBCT, see Figure 24. The voxels are cubes and their side is $0.2 \mathrm{~mm}$ long. The scan underwent a previous reshape in which empty pixels (corresponding to null Young's modulus) were added to the scan to form a image in order to fit the hierarchical mesh structure. We 


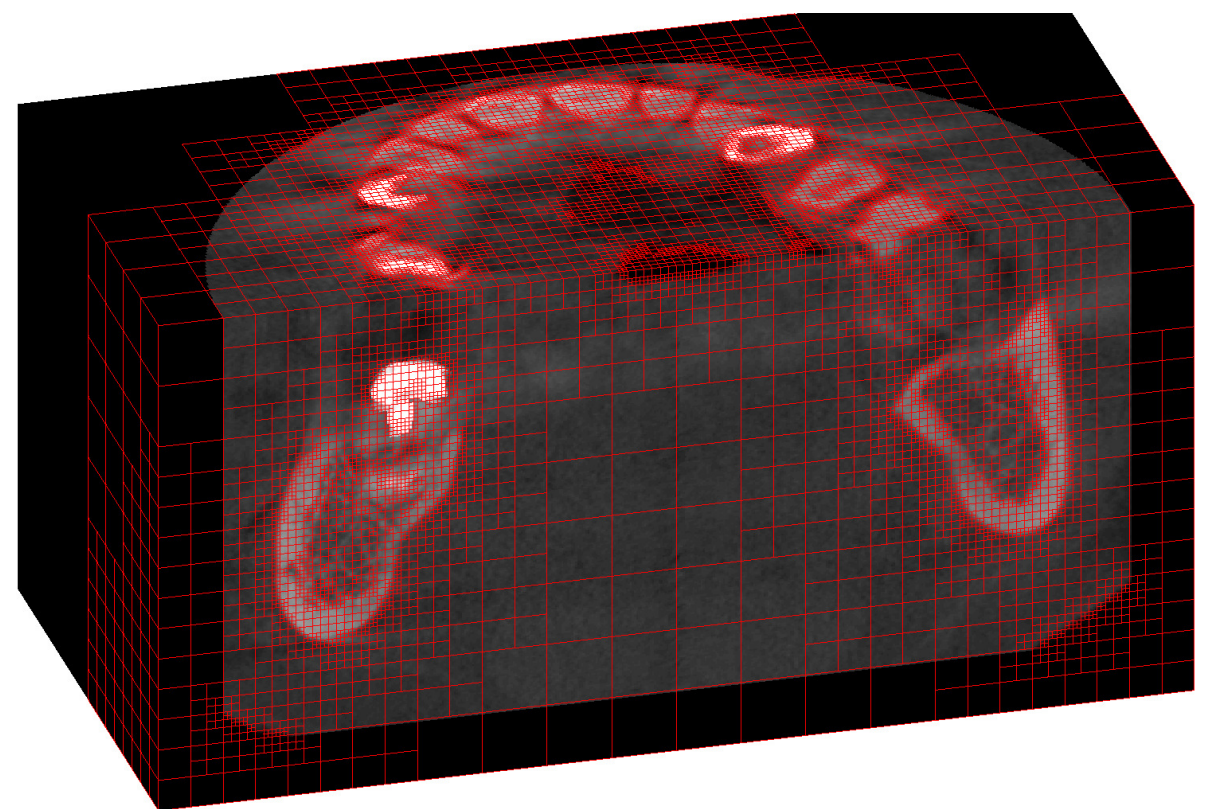

Figure 23: One eighth of a $h$-adapted mesh of an unsegmented dental CBCT scan.

\begin{tabular}{|l|l|l|l|l|l|l|}
\hline Grey level & 0 & 227 & 228 & 1500 & 2000 & 4095 \\
\hline E [GPa] & $10^{-4}$ & $10^{-4}$ & 0.5 & 1.5 & 7 & 30 \\
\hline
\end{tabular}

Table 4: Material Properties referred to Figure 19.

also applied a semi-automatic segmentation based on thresholding, domain growth and erosion which provided a logical matrix used to exclude from the calculation mesh elements only containing irrelevant voxels, see Figure 24 a).

Concerning the material properties, we use a fix Poisson's ratio of 0.3 whereas for the relationship between voxel value and Young's modulus a linear interpolation of the reference values in Table 4 . These were chosen from the literature without tackling the problem of the conversion between CBCT grey values and Hounfield scale, see Reeves et al. (2012), which exceeds the purpose of this paper.

The colour-based $h$-adapted mesh was obtained by setting the maximum Young's modulus range allowed in each element to $20 \%$ of the full-scale. The recursive refinement process was carried out on an initial uniform mesh coinciding with the level 2 of the nested Cartesian grid structure and the maximum level allowed for the elements was 8 , for which they contain $2 \times$ 


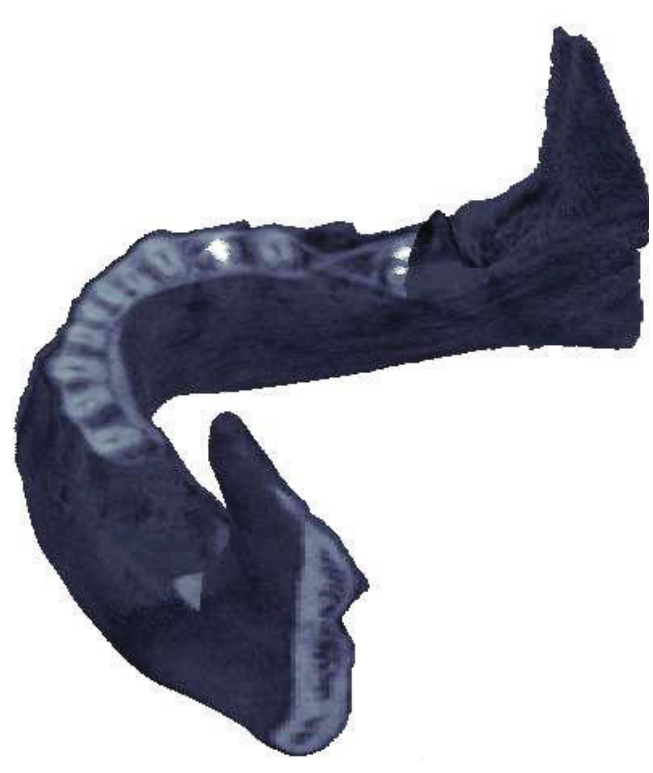

a)

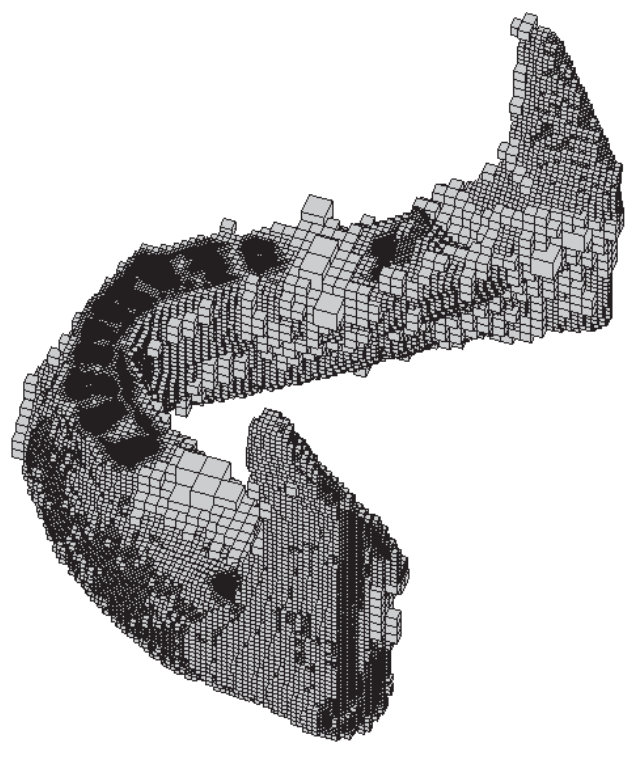

b)

Figure 24: Jaw CBCT scan based model a) Segmented jaw. b) FEAVox $h$-adapted mesh.

$2 \times 2$ voxels, see Figure $24 \mathrm{~b}$ ).

The element stiffness matrices were integrated by using LS-based technique, see Section 2.1. The boundary conditions of the problem are shown in Figure 25. All degrees of freedom were restrained in the nodes belonging to the green surface, whereas a uniform pressure of $1 \mathrm{MPa}$ is applied on the red square surface with a side $10 \mathrm{~mm}$ long.

The result is shown in Figure 26 in terms of von Mises stress field. The jaw bone cross section on the right lie on the vertical grey plane in Figure 25. In Figure 26 we can clearly distinguish the area where the Neumann boundary condition is applied and the bending stress distribution of the cortical bone on the jaw cross-section.

The model presented in this case study is obtained from a clinical CBCT scan and is characterised by the complexity and computational cost typical of actual simulation in the biomechanical field. Even though the software is implemented in MATLAB ${ }^{\circledR}$, which is affected by the typical slowness of 


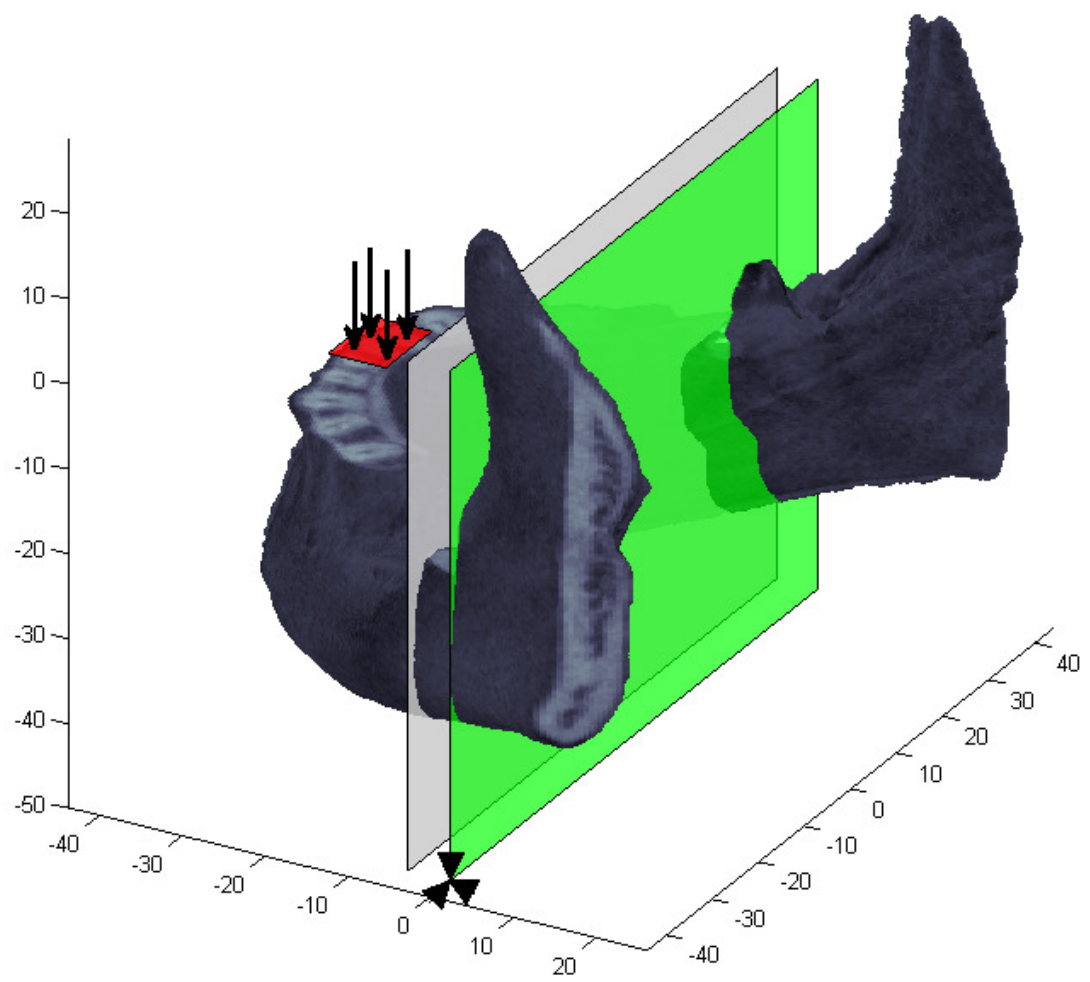

Figure 25: Boundary conditions for the problem in Figure 24. Lengths are expressed in $\mathrm{mm}$.

interpreted languages, it can handle problems of this size on a regular PC thanks to the characteristics of $c g$ FEM. In particular the mesh creation and the integration of the element stiffness matrices took advantage of the speed provided by the hierarchical Cartesian grid structure and the LS integration technique respectively whereas the computational cost of the solution of the system of equations is reduced by the DOF $h$-adaptive strategy and the DOF reordering made possible by the a priori knowledge of the mesh structure. This last aspect was exhaustively treated in Nadal (2014).

\subsection{Image and geometry-based cgFEM application. Dental implant}

The objective of this example is to test the suitability of FEAVox for the analysis of the interaction between a prosthesis and the surrounding biological tissue in the case of complete osteointegration following the procedure described in Section 2.3. For this purpose we use the 3D NURBs-based CAD 


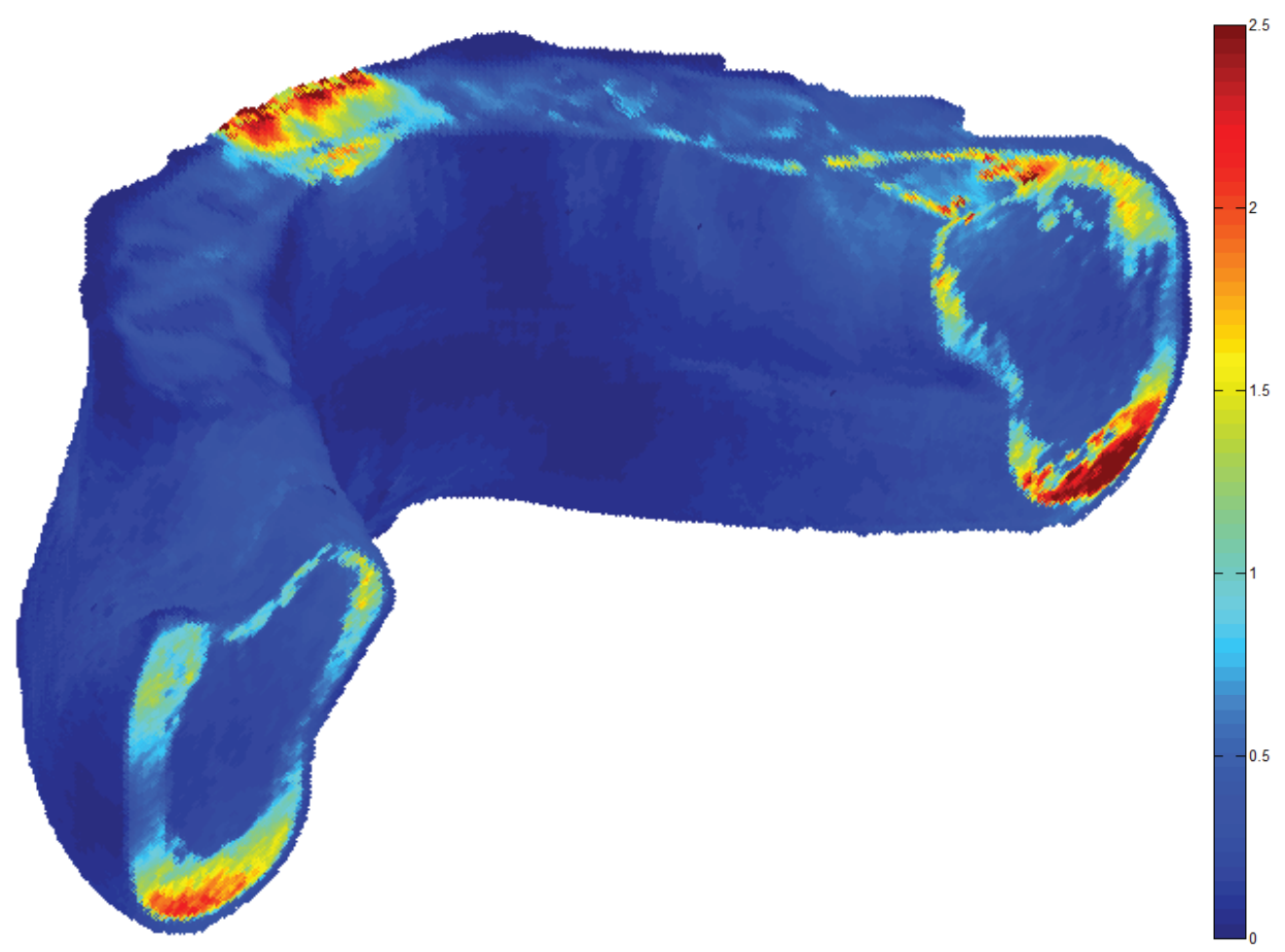

Figure 26: von Mises stress field expressed in MPa for the problem in Figure 24.

model of a dental implant, see Figure $27 \mathrm{~b}$ ) and the subregion highlighted in Figure 27 a) of the segmented jaw CBCT scan used for the simulation in Section 4.2.

The CAD model was immersed into the portion of the CBCT scan, the voxels contained in the device volume were deactivated in the corresponding logical sub-matrix from the segmentation process. The assembly and the corresponding $h$-adapted FE mesh are shown in Figure 28 a) and b) respectively. Homogeneous Dirichlet Boundary conditions are enforced upon all the DOFs of the nodes lying on the green surfaces in Figure 28 a) and a distributed load with components of $-2 \mathrm{MPa}$ in the $\boldsymbol{y}$ and $\boldsymbol{z}$ directions is applied on the CAD model surface highlighted in red. The bitmap and the geometry were initially immersed in a uniform mesh of level 4 and then an $h$-adaptive refinement was performed by imposing $\boldsymbol{I}_{\boldsymbol{R}}^{e} \leq 0.2$. The maximum refinement level allowed was 5 .

The same material properties as in the previous problem were used for the CBCT scan, see Section 4.2, except for the prosthesis which was assumed to 


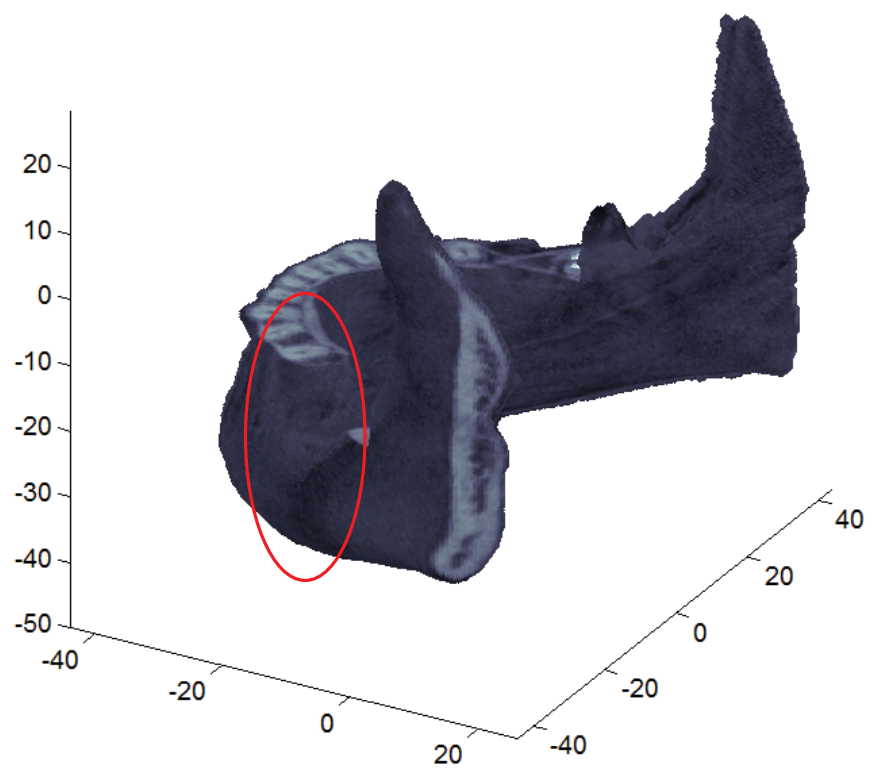

a)

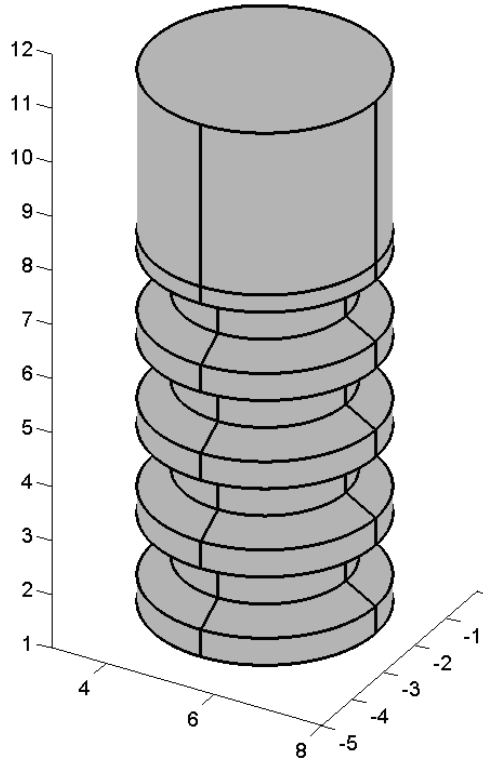

b)

Figure 27: Model components. Position is expressed in mm. a)Part of the CBCT ref used in this simulation b) Screw CAD model.

be made of titanium and, as a consequence, was assigned a Young's modulus and a Poisson's ratio of $110 \mathrm{GPa}$ and 0.32 respectively. The least-square integration scheme was used for the CBCT scan. In the elements cut by the CAD model the integration was performed by decomposition in tetrahedral subdomains on both sides of the interface, see Figure $11 \mathrm{~b}$ ). The subdomains on the interface were integrated by using the NEFEM-integration techniques as described by Marco et al. (2015).

Figure 29 a) shows the von Mises stress distribution of the assembly. In order to make visible the tress distribution inside the bone, the CBCT scan was cut with the grey plane shown in Figure 28 a) and only the front of the bitmap is shown. The von Mises stress distribution in the prosthetic device is magnified in Figure 29 b). The typical load transfer between the implant and the cortical bone at the implant upper threads and the lower stress state at the interface between the deep trabecular bone and the screw tip are visible. The stress map is plotted differently for the CBCT scan and and the CAD model. In the former the strain values are computed at the centre of the pixels and the $\mathbf{D}$ matrix associated to the pixel is used to compute the stress 


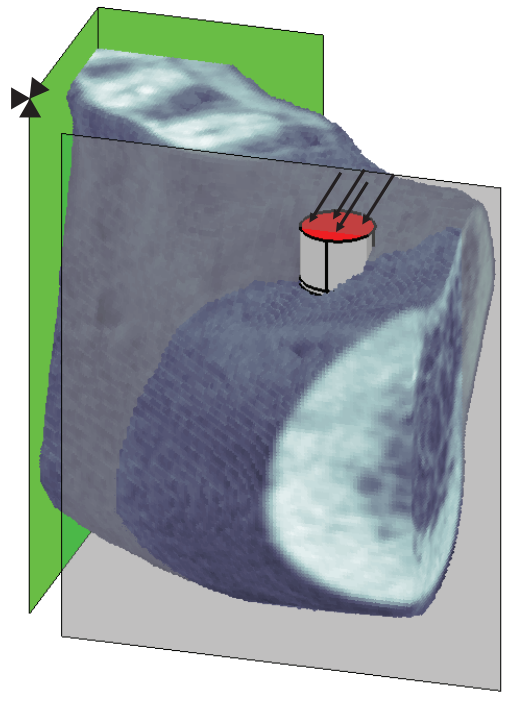

a)

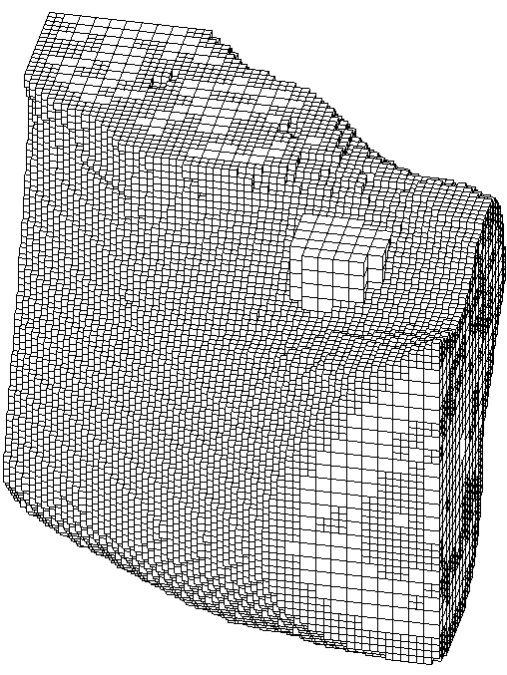

b)

Figure 28: Jaw CBCT scan based model a) Segmented jaw. b) FEAVox $h$-adapted mesh.

in that position. In the latter, in contrast, we use the SPR technique to obtain a $C_{0}$ continuous stress field. For representation purposes only, this is computed at the vertices of the external faces of the integration tetrahedra , represented as finer facets. This example shows how suitable $c g \mathrm{FEM}$ is for the simulation of osteointegrated implants in a patient specific framework. The method makes it possible to directly locate the implant CAD model on the volume of the bitmap model of the mandible to obtain a conjoined model. On the one hand, $c g$ FEM eliminates the necessity of further manipulation of the models and, on the other hand, it takes advantage of the specific strengths of each of them: the detailed spatial distribution and the elastic properties provided by the $\mathrm{CT}$ scans exact geometrical representation of the CAD.

\section{Conclusions}

This paper presents a special version of the Cartesian grid Finite Element Method ( $c g \mathrm{FEM})$ especially developed to solve image-based structural problems. 


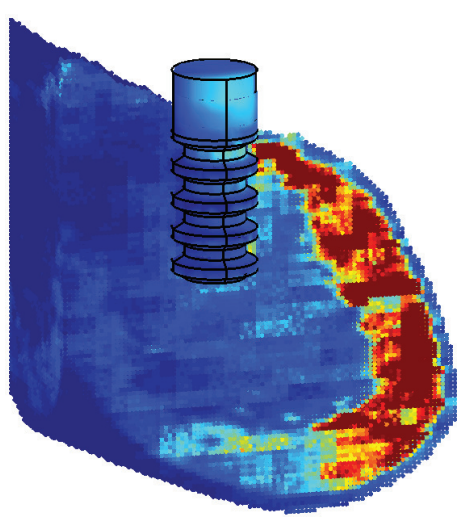

a)

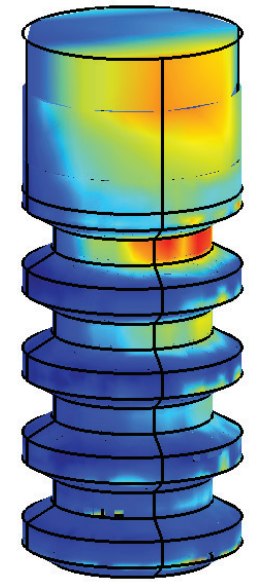

b)

Figure 29: von Mises stress field expressed in MPa. a) Front section of Figure 28 a). b) Prosthetic device detail.

It is based on a hierarchical structure of nested Cartesian grids and is capable of creating and refining FE models with very little user intervention taking into account the heterogeneity of the human tissues given by a medical image. The method is based on the availability of a reliable correlation between pixel values and material properties.

The most important characteristics of the method are: a) a reduction of the segmentation effort as long as voxel values are capable to account for the difference in the elastic behaviour (for instance, there is no need to separate the trabecular and cortical bone domains); b) the possibility of taking into account all the material property heterogeneity; c) a decrease of the geometrical modelling cost due to the fact that geometrical entities corresponding to the living tissues are only necessary for the imposition of the boundary conditions.

In addition, the method is suitable for modelling the effect of the interaction of implants with bones on the basis of preoperative medical images in a patient-specific framework. The methodology has been successfully implemented in 2 and 3D.

The validation tests justify the use of linear interpolation functions and 
indicate a proper refinement range in which the the modelling error is negligible and, therefore, the increase in the computational cost (mesh refinement) corresponds to a predictable increase in the solution accuracy. Two voxel-based and a least-square fitting-based integration schemes have been presented. The latter has proved to be as accurate as the other two in the framework $c g$ FEM $h$-adapted strategy while being computationally more efficient. The comparison between the reference and image-based models of different resolution shows an appropriate behaviour of the numerical technique.

This technique made it possible to easily create a FE model directly from an X-ray image and to obtain the bone stress distribution. Similarly, it allowed us to create a FE model of a system bone-prosthesis as a whole in a simple way. The numerical results obtained with X-ray and CBCT scans show the high potential of this technique.

\section{Acknowledgements}

With the support of the European Union Framework Programme (FP7) under grant agreement No. 289361 'Integrating Numerical Simulation and Geometric Design Technology (INSIST)', the Ministerio de Economía y Competitividad of Spain (DPI2010-20542) and the Generalitat Valenciana (PROMETEO/2016/007).

\section{Bibliography}

Alberich-Bayarri, A., Marti-Bonmati, L., Sanz-Requena, R., Belloch, E., Moratal, D., 2008. In vivo trabecular bone morphologic and mechanical relationship using high-resolution 3-T MRI. AJR. American journal of roentgenology 191 (3), 721-6.

Bah, M. T., Browne, M., Young, P. G., Bryan, R., Xuan, V. B., 2011. Effects of implant positioning in cementless total hip replacements. Computer Methods in Biomechanics and Biomedical Engineering 14 (sup1), 275-276.

Bordas, S. P. A., Rabczuk, T., Rodenas, J. J., Kerfriden, P., Moumnassi, M., Belouettar, S., 2010. Recent Advances Towards Reducing the Meshing and Re-Meshing Burden in Computational Sciences. Computational Technology Reviews 2, 51-82. 
Burman, E., Hansbo, P., 2010. Fictitious domain finite element methods using cut elements: I. a stabilized lagrange multiplier method. Computer Methods in Applied Mechanics and Engineering 199 (4144), 2680 - 2686.

Cottrell, J. A., Hughes, T. J. R., Bazilevs, Y., 2009. Isogeometric Analysis: Toward Integration of CAD and FEA, 1st Edition. Wiley.

Dance, D. R., Christofides, S., Maidment, A. D. A., McLean, I. D., Ng, K. H., 2014. Diagnostic Radiology Physics: A Handbook for Teachers and Students. IAEA, Vienna, Austria.

Dolbow, J., Harari, I., 4 2009. An efficient finite element method for embedded interface problems. International Journal for Numerical Methods in Engineering 78 (2), 229-252.

Grassi, L., Schileo, E., Taddei, F., Zani, L., Juszczyk, M., Cristofolini, L., 2012. Accuracy of finite element predictions in sideways load configurations for the proximal human femur. Journal of biomechanics 45 (2), 394-399.

Kim, H., Jürgens, P., Weber, S., Nolte, L.-P., Reyes, M., 2010. A new softtissue simulation strategy for cranio-maxillofacial surgery using facial muscle template model. Progress in biophysics and molecular biology 103 (2-3), 284-91.

Legrain, G., Cartraud, P., Perreard, I., Mos, N., 2011. An x-fem and level set computational approach for image-based modelling: Application to homogenization. International Journal for Numerical Methods in Engineering 86 (7), 915-934.

Liehr, F., Preusser, T., Rumpf, M., Sauter, S., Schwen, L. O., 2009. Composite finite elements for 3D image based computing. Computing and Visualization in Science 12 (4), 171-188.

Marco, O., Sevilla, R., Zhang, Y., Ródenas, J. J., Tur, M., 2015. Exact 3d boundary representation in finite element analysis based on cartesian grids independent of the geometry. International Journal for Numerical Methods in Engineering 103 (6), 445-468.

Müller-Karger, C. M., Rank, E., Cerrolaza, M., 2004. P-Version of the FiniteElement Method for Highly Heterogeneous Simulation of Human Bone. Finite Elements in Analysis and Design 40 (7), 757-770. 
Nadal, E., 2014. Cartesian Grid FEM (cgFEM): High Performance $h$ adaptive FE Analysis with Efficient Error Control. Application to Structural Shape Optimization. Ph.D. thesis, Dir. F. J. Fuenmayor, J. J. Ródenas, Universidad Politécnica de Valencia.

Nadal, E., Díez, P., Ródenas, J., Tur, M., Fuenmayor, F., 2015. A recoveryexplicit error estimator in energy norm for linear elasticity. Computer Methods in Applied Mechanics and Engineering 287, 172 - 190.

Nadal, E., Ródenas, J. J., Albelda, J., Tur, M., Tarancón, J. E., Fuenmayor, F. J., 2013. Efficient finite element methodology based on cartesian grids: Application to structural shape optimization. Abstract and Applied Analysis.

Natali, A. N., Pavan, P. G., Ruggero, A. L., 2006. Evaluation of stress induced in peri-implant bone tissue by misfit in multi-implant prosthesis. Dental materials : official publication of the Academy of Dental Materials 22 (4), 388-95.

Prez, M., Vendittoli, P.-A., Lavigne, M., Nuo, N., 2014. Bone remodeling in the resurfaced femoral head: Effect of cement mantle thickness and interface characteristics. Medical Engineering \& Physics 36 (2), 185 - 195.

Reeves, T., Mah, P., McDavid, W., 2012. Deriving hounsfield units using grey levels in cone beam ct: a clinical application. Dentomaxillofacial Radiology 41 (6), 500-508.

Roque, W. L., Arcaro, K., Alberich-Bayarri, A., 2012. Tortuosity and elasticity study of distal radius trabecular bone. In: Iberian Conference on Information Systems and Technologies, CISTI.

Ruess, M., Tal, D., Trabelsi, N., Yosibash, Z., Rank, E., 2012a. The finite cell method for bone simulations: verification and validation. Biomechanics and modeling in mechanobiology 11 (3-4), 425-37.

Ruess, M., Varduhn, V., Rank, E., Yosibash, Z., 2012b. A Parallel HighOrder Fictitious Domain Approach for Biomechanical Applications. 2012 11th International Symposium on Parallel and Distributed Computing, 279-285. 
Sutradhar, A., Park, J., Carrau, D., Nguyen, T. H., Miller, M. J., Paulino, G. H., 2016. Designing patient-specific 3d printed craniofacial implants using a novel topology optimization method. Medical \& Biological Engineering \& Computing 54 (7), 1123-1135.

Tur, M., Albelda, J., Marco, O., Ródenas, J., 2015. Stabilized method of imposing dirichlet boundary conditions using a recovered stress field. Computer Methods in Applied Mechanics and Engineering 296, 352-375.

Tur, M., Albelda, J., Nadal, E., Ródenas, J., 2014. Imposing dirichlet boundary conditions in hierarchical cartesian meshes by means of stabilized lagrange multipliers. International Journal for Numerical Methods in Engineering 98 (6), 399-417.

Viceconti, M., Bellingeri, L., Cristofolini, L., Toni, A., 1998. A comparative study on different methods of automatic mesh generation of human femurs. Medical engineering \& physics 20 (1), 1-10.

Yang, Z., Ruess, M., Kollmannsberger, S., Düster, A., Rank, E., 2012. An efficient integration technique for the voxel-based finite cell method. International Journal for Numerical Methods in Engineering 91 (June), 457471.

Zienkiewicz, O. C., Zhu, J. Z., 1987. A simple error estimator and adaptive procedure for practical engineering analysis. International Journal for Numerical Methods in Engineering 24 (2), 337-357. 